\title{
Spectro-photometric properties of the bulk of the radio-loud AGN population $\star$
}

\author{
R. D. Baldi ${ }^{1,2}$ and A. Capetti ${ }^{2}$ \\ ${ }^{1}$ Universitá di Torino, via P. Giuria 1, 10125 Torino, Italy \\ e-mail: baldi@oato.inaf.it \\ 2 INAF-Osservatorio Astronomico di Torino, Strada Osservatorio 20, 10025 Pino Torinese, Italy \\ e-mail: capetti@oato.inaf.it
}

Received 17 March 2010 / Accepted 18 May 2010

ABSTRACT

\begin{abstract}
In a previous paper we showed that the radio sources selected by combining large area radio and optical surveys, have a strong deficit of radio emission with respect to 3CR radio-galaxies matched in line emission luminosity. We argued that the prevalence of sources with luminous extended radio structures in high flux-limited samples is due to a selection bias. Sources with low radio power form the bulk of the radio-loud AGN population but are still virtually unexplored.

We here analyze their photometric and spectroscopic properties. From the point of view of their emission lines, the majority of the sample are Low Excitation Galaxies (LEG), similar to the 3CR objects at the same level of line luminosity. The hosts of the LEG are red, massive $\left(10.5 \lesssim \log M_{*} / M_{\odot} \lesssim 12\right)$ Early-Type Galaxies (ETG) with large black hole masses $\left(7.7 \lesssim \log M_{\mathrm{BH}} / M_{\odot} \lesssim 9\right)$, statistically indistinguishable from the hosts of low redshift 3CR/LEG sources. No genuine radio-loud LEG could be found associated with black holes with a mass substantially smaller than $10^{8} M_{\odot}$ or with a late type host. The fraction of galaxies with signs of star formation $(\sim 5 \%)$ is similar to what is found in both the quiescent ETG and 3CR/LEG hosts. We conclude that the deficit in radio emission cannot be ascribed to differences in the properties of their hosts. We argue that instead this could be due to a temporal evolution of the radio luminosity.

A minority $(\sim 10 \%)$ of the sample show rather different properties; these are associated with low black hole masses, with spiral galaxies, or with a high excitation spectrum. In general these outliers are the result of the contamination from Seyfert galaxies and from those where the radio emission is powered by star formation. For the objects with high excitation spectra there is no clear discontinuity in either the host or nuclear properties because they include radio-quiet as well as radio-loud AGN.
\end{abstract}

Key words. galaxies: active - galaxies: elliptical and lenticular, $\mathrm{cD}$ - galaxies: photometry - galaxies: jets

\section{Introduction}

The advent of large area surveys opened up the possibility for the scientific community to explore large samples of extragalactic sources and to set the results on several key issues on strong statistical foundations. The cross-match of astronomical data from radio and optical surveys specifically provides a unique tool in the analysis of the radio-emitting galaxies, because it allows us to identify optically large numbers of radio sources, to obtain spectroscopic redshifts, to determine the properties of their hosts, and to build up their spectral energy distributions.

In recent years several studies have indeed been carried out on large samples of radio galaxies in order to investigate the links between the radio structures, the central engine associated with an Active Galactic Nucleus (AGN), and the host galaxies. In particular, Best et al. (2005a) selected a sample of 2215 low-luminosity radio galaxies by cross-correlating SDSS (Data Release 2), NVSS, and FIRST ${ }^{1}$. The resulting catalog is

* Appendix is only available in electronic form at http: //www . aanda . org

1 Sloan Digital Sky Survey, (York et al. 2000), National Radio Astronomy Observatory (NRAO) Very Large Array (VLA) Sky Survey (Condon et al. 1998), and the Faint Images of the Radio Sky at Twenty centimeters survey (Becker et al. 1995) respectively. by far more extensive than any previously studied sample of fully optically identified radio-sources. This sample (hereafter we refer to it as SDSS/NVSS sample) is highly (95\%) complete down to the flux threshold of $5 \mathrm{mJy}$ and provides a very good representation of radio-galaxies in the local Universe, up to a redshift of $\lesssim 0.3$, covering the range $10^{38}-10^{42} \mathrm{erg} \mathrm{s}^{-1}$ in radio power.

In a subsequent paper (Best et al. 2005b) studied the properties of the host galaxies of these radio-loud AGN (hereafter RLAGN), finding that these are massive galaxies, usually of the early Hubble type, with stellar masses in the range log $\left(M_{*} / M_{\odot}\right) \sim 10^{10}-10^{12}$, and located in richer environments than normal galaxies.

The SDSS/NVSS sample was then considered by Baldi \& Capetti (2009) in the context of the properties of low-luminosity radio-galaxies. We show that they display a strong deficit of radio emission with respect to their nuclear emission-line luminosity when compared to sources, part of other samples of radiogalaxies, matched in line luminosity.

This result is particularly intriguing considering that a strong correlation between line and radio luminosity is well established (see, e.g., Baum \& Heckman 1989; Rawlings et al. 1989; Morganti et al. 1997; Tadhunter et al. 1998; Willott et al. 1999) from the study of different samples of radio-galaxies. Both Low 
and High Excitation Galaxies (LEG and HEG respectively) separately obey this correlation, although the normalizations for the two classes differ (Buttiglione et al. 2010). This suggests that the energy source of the narrow lines is closely linked to the source of the radio emission. Conceivably a constant fraction of the available accretion power is channeled into radiative emission (that powers the emission line regions) and into jet kinetic energy (of which the radio emission is the electromagnetic manifestation). The study of the SDSS/NVSS sample suggests instead that total radio luminosity and line emission are independent of each other.

Nonetheless, considering the properties of miniature radiogalaxies (Balmaverde \& Capetti 2006) we found that a link exists between radio core and line emission, extending the same relation present in high power sources to low luminosities. By assuming that the [O III] luminosity gives an appropriate bolometric estimate of the AGN power and that radio cores are a good proxy for the jet power, radio galaxies with similar nuclear properties are able to produce an extremely wide range of total radio power, of a factor $\geq 100$. Indeed, these radio-galaxies of a very low power are all highly core-dominated, with only a feeble extended emission. A similar sample, studied by Prandoni et al. (2010) at higher frequency $(1.4-15 \mathrm{GHz})$ and selected at lower flux cutoff $(>1 \mathrm{mJy})$, also shows a substantial fraction of very compact radio morphologies $(d<10 \mathrm{kpc})$ in the same range of radio power covered by $3 \mathrm{CR}$ and $\mathrm{B} 2$ sample. We argued that the prevalence of sources with luminous extended radio structures in high flux limited samples is due to a selection bias, because the inclusion of these objects is highly favored. Core-dominated sources with a low ratio between radio and emission line power form the bulk of the local radio-loud AGN population, but they are still virtually unexplored. Unfortunately, the images available for the SDSS/NVSS sample are not sufficient to properly isolate their radio cores.

The aim of this paper is a better understanding of the physical properties of these RLAGN. In particular we will explore the spectro-photometric properties of their hosts looking for possible differences that might explain their low level of radio emission with respect to the classical powerful radio galaxies matched in line luminosity. Operatively, with the information provided by SDSS we classify the optical spectra of the AGN on the basis of the excitation level. Then we analyze the key properties of the hosts and the AGN, such as the black hole mass, the mass and colors of the hosts, drawing a quantitative comparison with the $3 \mathrm{CR}$ sample. The main result is that the sample shows indistinguishable properties from those of 3CR sample. This implies that the reasons for the different radio properties between the bulk of the RLAGN population and the most studied sample of radio-galaxies must be ascribed to other mechanisms.

The paper is organized as follows. In Sect. 2 we briefly present the sample selection carried out by Best et al. (2005b). In Sects. 3 and 4 we analyze the spectro-photometric properties of the SDSS/NVSS sample considering separately the subsamples in the redshift ranges $0.03<z<0.1$ and $0.1<z<0.3$. What emerges from the study is that the sample is mostly composed of red, massive early-type galaxies with a large black hole mass, with spectra typical of LEG. In Sect. 5 we compare their broadband properties with those of the 3CR sample, finding a clear similarity with LEG. The few apparent exceptions to this general rule are discussed in Sect. 6. The summary and conclusions to our findings are given in Sect. 7. We provide the spectrophotometric diagrams of the sub-sample with $0.1<z<0.3$ in the Appendix.

\section{Sample selection}

For the analysis we used the sample of RLAGN selected by Best et al. (2005b). This sample is obtained cross-matching the 212000 galaxies drawn from the SDSS-DR2 with the NVSS and FIRST radio surveys. Leaving aside 497 sources identified as star-forming galaxies, this radio-selected sample consists of 2215 radio luminous AGN with a radio flux higher than $5 \mathrm{mJy}$ at $1.4 \mathrm{GHz}$.

The Sloan Digital Survey (York et al. 2000; Stoughton et al. 2002) with its optical imaging and spectroscopic survey of about a quarter of the sky provides a variety of physical parameters for the galaxies of this sample. These include emission line fluxes, stellar velocity dispersions, optical magnitudes in five bands, stellar masses, concentration indices, and strength of stellar absorption features such as the $4000 \AA$ break strength $\left(D_{n}(4000)\right)$. These photometric and spectroscopic parameters have been used for the present analysis.

\section{Results: the sub-sample with $0.03<z<0.1$}

In this section we describe the results obtained considering the sub-sample of 425 SDSS/NVSS galaxies with $0.03<z<0.1$. The more distant objects will instead be discussed in Sect. 4.

\subsection{Optical spectroscopic diagnostic diagrams}

Kewley et al. (2006) considered a sample of $~ 85000$ emission line galaxies from the SDSS, using the location of a galaxy in the spectroscopic diagnostic diagrams, planes formed by pair of emission line ratios, to recognize the nature of their nuclear emission, e.g. separating active nuclei from star-forming galaxies (e.g. Baldwin et al. 1981). Furthermore, Kewley et al. (2006) noted that AGN form branches of different excitation level, i.e. Seyfert and LINER (Heckman 1980).

We start our analysis considering the 425 objects with $0.03<$ $z<0.1$. In the first three panels of Fig. 1 we show the location in the diagnostic diagrams for the objects with emission lines detected at $S N R>3$ separately for each diagram. In the plane $\log ([\mathrm{O} \mathrm{III}] / \mathrm{H} \beta)$ vs. $\log ([\mathrm{O} \mathrm{I}] / \mathrm{H} \alpha)$ there is a well defined "branch" of low excitation galaxies. We also find high excitation AGN, but only very few.

Best et al. (2005b) excluded galaxies in which the radio emission can be accounted for star formation ${ }^{2}$. Nonetheless, several objects with a starburst-like optical spectrum are included in the sample. The radio emission in these galaxies is possibly powered by the AGN, but their emission lines likely have a dominant contribution from regions of star formation.

Our optical classification is based on the simultaneous identification of an object in all three diagnostic diagrams. 212 objects, with a $S N R>3$ for each of the six key emission lines, could be classified (see Table 1). A source is classified as Star-Forming Galaxy (SFG), when it is located below the curved solid lines in even just one diagram. This approach is rather conservative and can lead to an overestimate of the number of SFG. LEG (HEG) are the sources located in the LINER (Seyfert) region in both [S II]/H $\alpha$ and $[\mathrm{O} \mathrm{I}] / \mathrm{H} \alpha$ planes; an "ambiguous" galaxy (AG) changes its identification from LEG to HEG (or vice-versa) in the diagrams involving the $[\mathrm{S} \mathrm{II}] / \mathrm{H} \alpha$ and

\footnotetext{
2 Best et al. (2005b) divided the sample into two classes: RLAGN and galaxies in which the radio emission is dominated by star formation. The division is based on the location of a galaxy in the plane of 4000- $\AA$ break strength versus radio luminosity per unit stellar mass, see Fig. 6.
} 
R. D. Baldi and A. Capetti: Spectro-photometric properties of the radio-loud AGN population

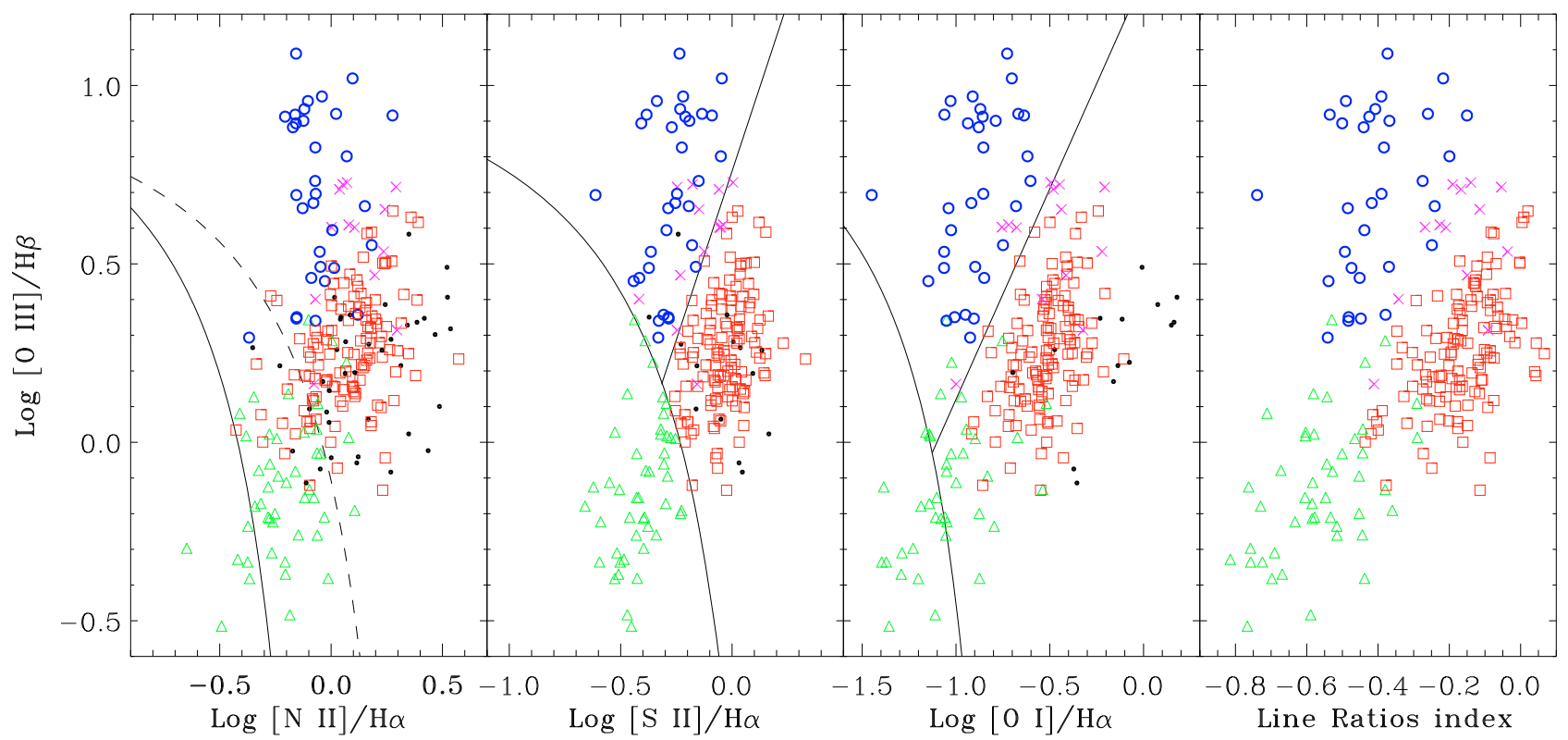

Fig. 1. Spectroscopic diagnostic diagrams for the objects with $0.03<z<0.1$ : in the first panel, $\log ([\mathrm{O} \mathrm{III}] / \mathrm{H} \beta)$ vs. $\log ([\mathrm{N}$ II $] / \mathrm{H} \alpha)$, in the second $\log ([\mathrm{O} \mathrm{III}] / \mathrm{H} \beta)$ vs. $\log ([\mathrm{S} \mathrm{II}] / \mathrm{H} \alpha)$, and in the third, $\log ([\mathrm{O} \mathrm{III}] / \mathrm{H} \beta)$ vs. $\log ([\mathrm{O} \mathrm{I}] / \mathrm{H} \alpha)$. The solid lines separating star-forming galaxies, LINER, and Seyfert galaxies are from Kewley et al. (2006); in the first panel the region between the two curves is populated by the composite galaxies. In the fourth panel we give the location of each source derived from the previous diagrams in the plane $\log ([\mathrm{O} \mathrm{III}] / \mathrm{H} \beta)$ vs. line ratios index (see text). We mark LEG as red squares, HEG as blue circles, star-forming Galaxies as green triangles, ambiguous galaxies as pink crosses, and unclassified galaxies as black dots.

Table 1. Spectral and morphological classification breakdown

\begin{tabular}{cccccccc}
\hline \hline & & Unclass. & LEG & AG & HEG & SFG & Total \\
$0.03<z<0.1$ & & $\mathbf{2 1 3}$ & $\mathbf{1 2 2}$ & $\mathbf{1 3}$ & $\mathbf{3 2}$ & $\mathbf{4 5}$ & $\mathbf{4 2 5}$ \\
\hline$C_{r}>2.86$ & $D_{n}(4000)>1.7$ & 181 & 88 & 9 & 4 & 8 & 290 \\
and $\sigma\left(C_{r}\right)<0.2$ & $D_{n}(4000)<1.7$ & 1 & 7 & 1 & 11 & 9 & 29 \\
& subtotal & $\mathbf{1 8 2}$ & $\mathbf{9 5}$ & $\mathbf{1 0}$ & $\mathbf{1 5}$ & $\mathbf{1 7}$ & $\mathbf{3 1 9}$ \\
$2.6<C_{r}<2.86$ & $D_{n}(4000)>1.7$ & 14 & 9 & 0 & 1 & 3 & 27 \\
and $\sigma\left(C_{r}\right)<0.2$ & $D_{n}(4000)<1.7$ & 0 & 4 & 0 & 8 & 8 & 20 \\
& subtotal & $\mathbf{1 4}$ & $\mathbf{1 3}$ & $\mathbf{0}$ & $\mathbf{9}$ & $\mathbf{1 1}$ & $\mathbf{4 7}$ \\
$C_{r}<2.6$ & $D_{n}(4000)>1.7$ & 2 & 3 & 2 & 0 & 2 & 9 \\
and $\sigma\left(C_{r}\right)<0.2$ & $D_{n}(4000)<1.7$ & 2 & 4 & 1 & 6 & 13 & 26 \\
& subtotal & $\mathbf{4}$ & $\mathbf{7}$ & $\mathbf{3}$ & $\mathbf{6}$ & $\mathbf{1 5}$ & $\mathbf{3 5}$ \\
Total & & $\mathbf{2 0 0}$ & $\mathbf{1 1 5}$ & $\mathbf{1 3}$ & $\mathbf{3 0}$ & $\mathbf{4 3}$ & $\mathbf{4 0 1}$ \\
$C_{r}>2.86$ & "red" & 176 & 87 & 9 & 6 & 11 & $\mathbf{2 8 9}$ \\
and $\sigma\left(C_{r}\right)<0.2$ & "blue" & 6 & 8 & 1 & 9 & 6 & $\mathbf{3 0}$ \\
\hline
\end{tabular}

[O I] $/ \mathrm{H} \alpha$ ratios. The sub-sample with spectroscopic classification is mostly composed of LEG (122), while there are 32 HEG, $45 \mathrm{SFG}$, and $13 \mathrm{AG}$. The remaining half of the sample $(213 \mathrm{ob}-$ jects) has no optical classification. Their properties will be discussed in more detail below.

We also estimated the line ratios index (LRI) for our sources, the new spectroscopic indicator introduced by Buttiglione et al. (2010). This is defined as the average of the low ionization lines ratios, i.e. $1 / 3(\log [\mathrm{N} \mathrm{II}] / \mathrm{H} \alpha+\log [\mathrm{S} \mathrm{II}] / \mathrm{H} \alpha+\log [\mathrm{O} \mathrm{I}] / \mathrm{H} \alpha)$ and provides a more stable classification than the individual line ratios. In the fourth panel of Fig. 1 we show the location of the sources in the $[\mathrm{O} \mathrm{III}] / \mathrm{H} \beta$ vs. LRI plane, distinguished according to the optical classification derived above. Two populations of low/high excitation level AGN are clearly seen, similar to that observed in the previous diagnostic diagrams and for the RLAGN sample of 3CR radio-galaxies (Buttiglione et al. 2010). As expected, the ambiguous galaxies are located in an intermediate region between LEG and HEG, but mostly at high $[\mathrm{O}$ III $] / \mathrm{H} \beta$ values.

While the sample analyzed here is radio selected, the active nuclei considered in Kewley et al. (2006) are mostly radioquiet. Therefore it is appropriate to also consider the results of Buttiglione et al. (2010) on the spectroscopic properties of radio galaxies from the 3CR sample. Following previous studies, (e.g. Hine \& Longair 1979; Laing et al. 1994; Jackson \& Rawlings 1997), they separated low and high excitation galaxies (LEG and HEG respectively) on the basis of the narrow emission line ratios. The spectroscopic separation of RLAGN into HEG/LEG slightly differs from the radio-quiet Seyfert/LINER classification. Indeed, the branch of LEG reaches [O III] $/ \mathrm{H} \beta$ ratios $\sim 0.2$ dex higher than the LINER from Kewley et al. (2006). It is unclear whether this is due to a luminosity mismatch (the 3CR line luminosity is on average $\sim 30$ times higher than the SDSS sources) or to a genuine difference between radio-quiet 

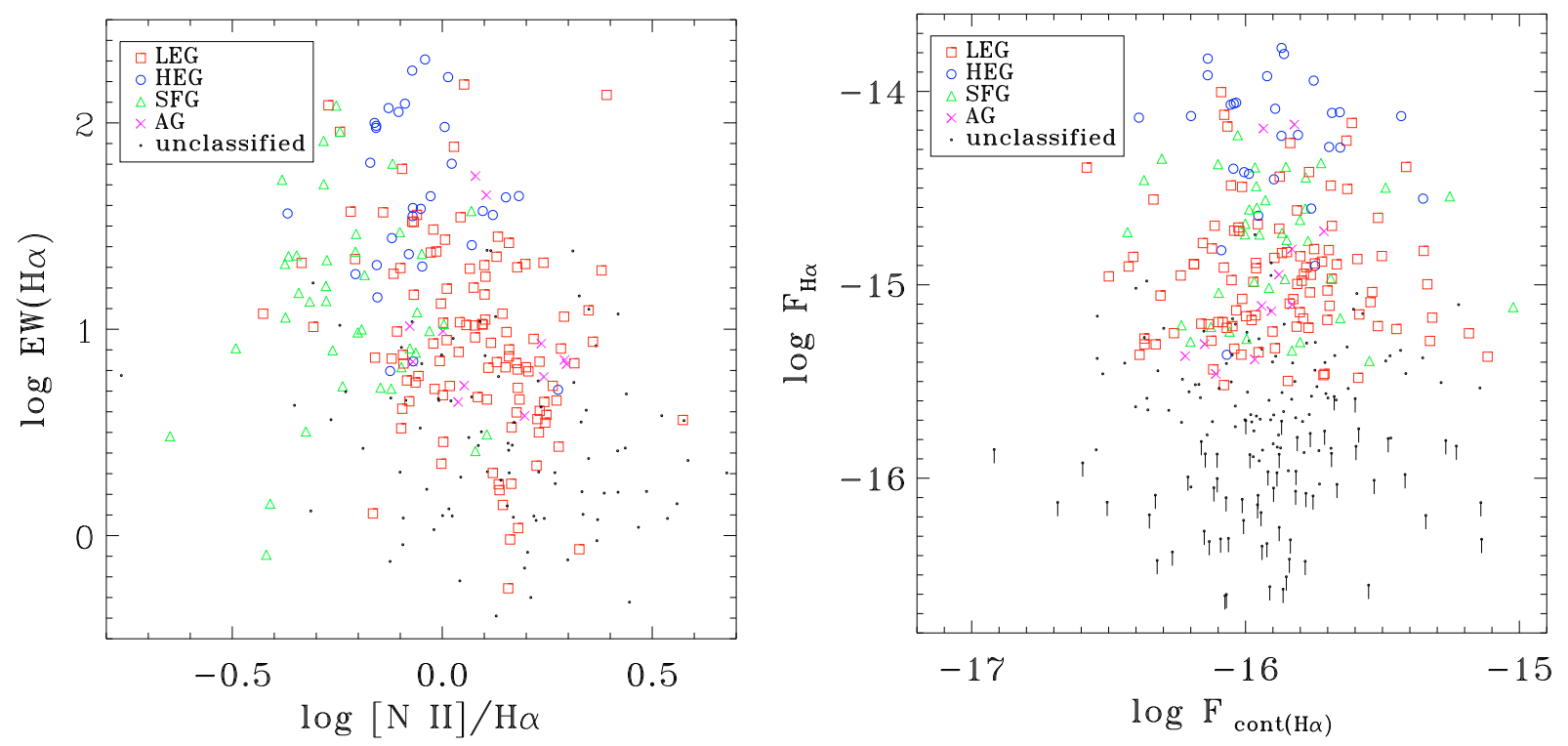

Fig. 2. Left: $\mathrm{H} \alpha$ equivalent width against the $[\mathrm{N}$ II]/H $\alpha$ ratio. Only objects with both [N II] and $\mathrm{H} \alpha$ detected are shown. Right: logarithm of the $\mathrm{H} \alpha$ flux versus logarithm of the continuum at the $\mathrm{H} \alpha$ line (in $\mathrm{erg} \mathrm{s}^{-1}$ and $\mathrm{erg} \mathrm{s}^{-1} \AA^{-1}$ units respectively).

and RLAGN in the diagnostic diagrams. Because the objects considered here have line luminosities similar to the Kewley et al. (2006) sources (most of our objects have $10^{39}<L_{[\mathrm{O} \text { III] }}<$ $3 \times 10^{40}$ ), we decided to adopt their classification of High and Low excitation galaxies. We will however bear in mind that with this choice objects located just above the Seyfert/LINER separation lines could instead be genuine low excitation RLAGN.

Let us now focus on the objects that have no optical classification (hereafter unclassified galaxies, UG) because at least one of the key emission lines (usually $\mathrm{H} \beta$ and/or [O III]) is undetected. This subsample is clearly very important because it is composed of 213 objects, about half of the low redshift galaxies $^{3}$. First of all, $\sim 50$ such objects can still be positioned in at least one of the diagnostic diagrams of Fig. 1. With only two possible exceptions, they fall into the same region as populated by LEG.

Cid Fernandes et al. (2010) showed that a good separation between the various spectroscopic classes can also be obtained by comparing the $\mathrm{H} \alpha$ equivalent width $(E W)$ and the $[\mathrm{N} \mathrm{II}] / \mathrm{H} \alpha$ ratio (see Fig. 2). In the SDSS/NVSS sample, HEG generally have the highest values of $E W(\mathrm{H} \alpha)$. SFG and LEG span a similarly broad range of $E W(\mathrm{H} \alpha)$, but these two classes are still rather well separated, particularly at low equivalent widths, where the SFG have lower values of $[\mathrm{N} \mathrm{II}] / \mathrm{H} \alpha$ than LEG. The 116 UG present in this diagnostic diagram are located in the same region as the LEG with only a few possible exceptions and cluster in the lower right corner. This also applies to the UG with only [N II] detection. This strengthens the idea that the unclassified objects belong to the class of LEG, but with an even lower contrast of the AGN against the host galaxy emission. In the right panel of Fig. 2 we instead compare the $\mathrm{H} \alpha$ flux and the continuum level around this line. This shows that UG are located at low $\mathrm{H} \alpha$ fluxes, indicating that their lower $E W(\mathrm{H} \alpha)$ is due to a lower line flux and not to a higher continuum level.

\footnotetext{
3 Also in the 3CR sample $29(28 \%)$ galaxies with $z<0.3$ cannot be properly identified spectroscopically (Buttiglione et al. 2009, 2010), and they are almost as numerous as the LEG (32).
}

\subsection{Spectro-photometric diagnostic diagrams}

We now investigate the spectro-photometric properties of the host galaxies, taking advantage of the information about their optical spectroscopic classification. We will then consider the host morphology, the stellar age and the content of young stars, the broad band colors, their mass, and the mass of their central supermassive black hole (SMBH).

Among the variety of measures provided by SDSS, the concentration index $C_{r}$ (defined as the ratio of the radii including $90 \%$ and $50 \%$ of the light in the $r$ band respectively) can be used for a morphological classification of the hosts. Early-type galaxies (hereafter ETG) have higher values of $C_{r}$. Two thresholds have been suggested to define ETG: a more conservative value at $C_{r} \geq 2.86$ (e.g. Nakamura et al. 2003; Shen et al. 2003) and a more relaxed selection at $C_{r} \geq 2.6$ (e.g. Strateva et al. 2001; Kauffmann et al. 2003; Bell et al. 2003). Bernardi et al. (2010) found that the second threshold of the concentration index corresponds to a mix of $\mathrm{E}+\mathrm{S} 0+\mathrm{Sa}$ types, while the first mainly selects elliptical galaxies, removing the majority of Sa's, but also some Es and S0s. We select from the previously spectroscopically selected sample only the objects with an r-band concentration index measured with an accuracy better than $\sigma\left(C_{r}\right)<0.2$. It is composed of 401 objects (see Table 1): most of them (80\%) are secure ETG (with $C_{r}>2.86$ ), while only a small fraction $(9 \%)$ are most likely late-type galaxies (with $C_{r}<2.6$ ). The remaining $11 \%$ of the sub-sample are located in the range $2.6<C_{r}<2.86$.

Another important parameter is the $4000 \AA$ break strength, $D_{n}(4000)$ (defined as the ratio between the fluxes in the wavelength range $3850-3950 \AA$ and $4000-4100 \AA$ ), which is sensitive to the presence of a young stellar population (Balogh et al. 1999). But it is insensitive to the effects of the internal reddening, unlike broad-band colors. The distribution of $D_{n}(4000)$ in the large sample studied by Kauffmann et al. (2003) is bimodal, with massive galaxies $\left(\log M_{*} / M_{\odot} \gtrsim 10.5\right)$ showing a larger break than less massive objects. The transition between the two groups is located approximately at $D_{n}(4000) \sim 1.7$.

Figure 3 shows the distribution of the RLAGN in the spectrophotometric diagram composed of $C_{r}$ vs. $D_{n}(4000)$. Most of the points are located in the region of the plane populated by 
R. D. Baldi and A. Capetti: Spectro-photometric properties of the radio-loud AGN population

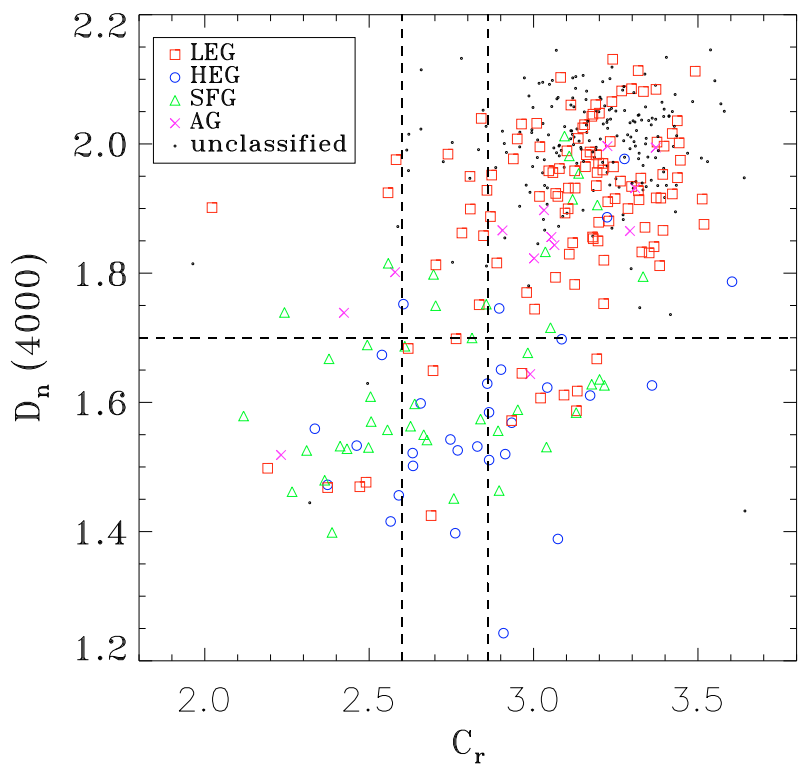

Fig. 3. Concentration index in $r$ band, $C_{r}$, vs. $4000 \AA$ break, $D_{n}(4000)$, for the 401 objects of the SDSS/NVSS AGN sample with $0.03<z<0.1$ and error on the $r$-band concentration index $<0.2$.

quiescent ETG. These are predominantly LEG and unclassified objects. The objects with $D_{n}(4000)<1.7$ are broad over a large range of $C_{r}$, encompassing both early and late-type galaxies. All spectroscopic classes are well represented in this region of the plane, but with a dominant contribution of SFG and HEG.

Let us now consider the location of the different spectroscopic classes. The majority of LEG are hosted in quiescent $\left(D_{n}(4000)>1.7\right)$ ETG $\left(76 \%\right.$ for $C_{r}>2.86$ and $84 \%$ for $\left.C_{r}>2.6\right)$ and this behavior is even more pronounced in the spectroscopically unclassified sources (with percentages of $90 \%$ and $98 \%$ respectively). Only a small fraction (13\% among LEG and $2 \%$ among UG) of these two classes show a possible evidence for star formation, with $D_{n}(4000)<1.7$. Similarly, only $7 \%$ of the LEG and $2 \%$ UG likely have late-type hosts. The ambiguous galaxies are also mostly red and quiescent.

The situation is radically different for SFG and HEG. They cover the whole range of values for $C_{r}$ and preferentially show low values of $D_{n}(4000): 83 \%$ of the HEG and $70 \%$ of the SFG have $D_{n}(4000)<1.7$.

The color index $u-r$ is another parameter able to qualify the star-formation rate of the host as a whole. Indeed, while the $D_{n}(4000)$ index is measured over the $3^{\prime \prime}$ SDSS fiber centered on the nucleus, the $u-r$ color is estimated over the entire galaxy. In Fig. 4 we show the $u-r$ color vs. the absolute $r$-band magnitude $M_{r}$ of the hosts of the sample limiting to the secure ETG, i.e. $C_{r}>2.86$. Most of the sample is located at color $u-r>2.5$ and $M_{r}<-21$. However, a tail of bluer galaxies is present, formed mostly by HEG and SFG, but including also a few LEG and UG (see Table 1 for a summary). On average, UG are redder and brighter than LEG.

In Fig. 4 the dashed line represents the separation between "red" and "blue" galaxies introduced by Schawinski et al. (2009) for a volume-limited SDSS sample $(0.02<z<0.05)$ of ETG, classified morphologically by visual inspection by the Galaxy Zoo project (Lintott et al. 2008). This sample shares the luminosity range, $M_{r}<-20.7$ with our radio-selected sample, straddling the $M_{*}$ for low-redshift ETG $\left(M_{r, *}=-21.15\right.$, Bernardi et al. 2003). With the aim of isolating ETG with ongoing star formation, Schawinski et al. (2009) determined the $3 \sigma$ offset from

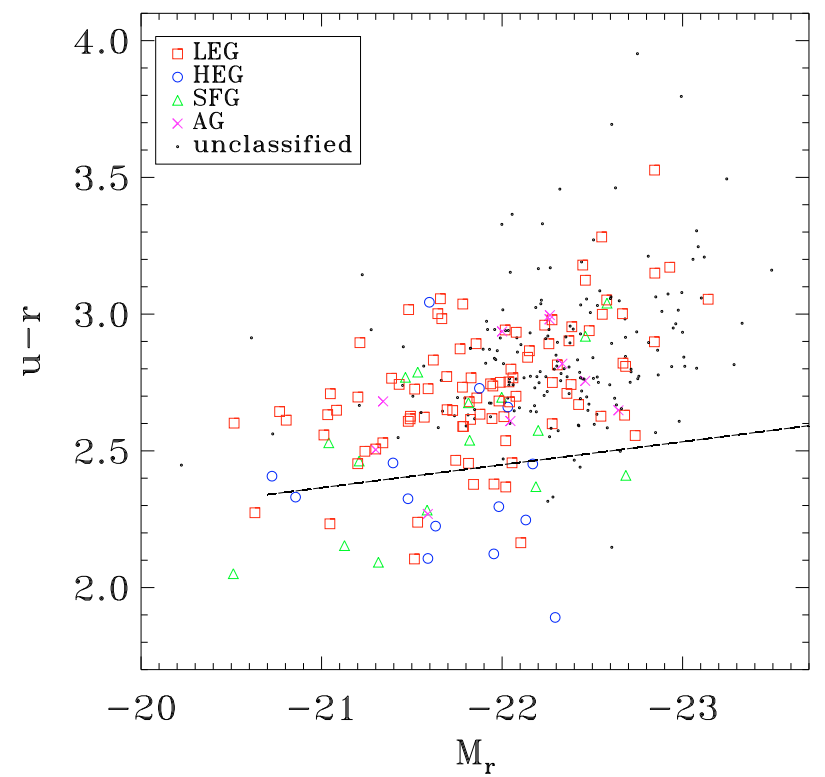

Fig. 4. Absolute $r$ band magnitude, $M_{r}$, vs. $u-r$ color for the sample of secure ETG (i.e. with $C_{r}>2.86$ ) with $0.03<z<0.1$. The dashed line separates the "blue" ETG from the red sequence, following the definition of Schawinski et al. (2009).

the mean of the red sequence in $u-r$ vs. $M_{r}$ plane and defined as blue ETG those that are below this threshold.

LEG and UG typically do not show sign of an ongoing star formation. Indeed, the fraction of these galaxies with blue $u-r$ color is $\sim 5 \%$ ( $3 \%$ of the UG and $8 \%$ of the LEG). In the sample considered by (Schawinski et al. 2009) the observed fraction of "blue" ETG is $5.7 \%$. This indicates that the fraction of star-forming galaxies ${ }^{4}$ in UG and LEG in our radioselected sample is similar to that of the general population of ETG. Furthermore, the fraction of "blue" ETG decreases with luminosity, and they disappear in galaxies with $M_{r}<-22.5$ in agreement with the results by Schawinski et al. (2009).

Conversely, HEG show a high fraction $(60 \%)$ of blue objects and, not unexpectedly, also SFG are often (35\%) blue.

SDSS also provides us with the stellar velocity dispersion of the sample, from which we derive the SMBH masses $\left(M_{\mathrm{BH}}\right)$ adopting the relation of Tremaine et al. (2002). To better visualize the $M_{\mathrm{BH}}$ distribution for each class of host galaxies and AGN, we show in Fig. 5 the SMBH mass against the concentration index. The vast majority of LEG and UG is associated with ETG (as already shown before) with large SMBH masses $\left(8 \lesssim \log \left(M_{\mathrm{BH}} / M_{\odot}\right) \lesssim 9\right)$. A tail of later-type galaxies extends toward smaller SMBH masses (reaching $\log \left(M_{\mathrm{BH}} / M_{\odot}\right) \sim 6.5$ ) and is formed mostly by HEG and SFG.

Let us explore the origin of the radio emission and in particular the contamination related to star formation. The criterion applied by Best et al. (2005b) to exclude radio sources powered (or strongly influenced) by star formation is based on the location of a galaxy in the $D_{n}(4000)$ versus $L_{1.4 \mathrm{GHz}} / M_{*}$ plane, where $M_{*}$ is the galaxy's stellar mass ${ }^{5}$. This allows one to predict the radio emission per unit mass expected from a stellar population of a given age. As seen in Fig. 9 of Best et al. (2005b),

\footnotetext{
${ }^{4}$ Note also that in our sample there is the possibility that the blue color could be related to a substantial contribution of the AGN continuum.

${ }_{5} M_{*}$ is estimated from the $g$ and $z$ SDSS magnitudes following following Bell et al. (2007) resulting in slightly different masses from those reported by Best et al. (2005b). This explains that some sources are located below the threshold curve.
} 


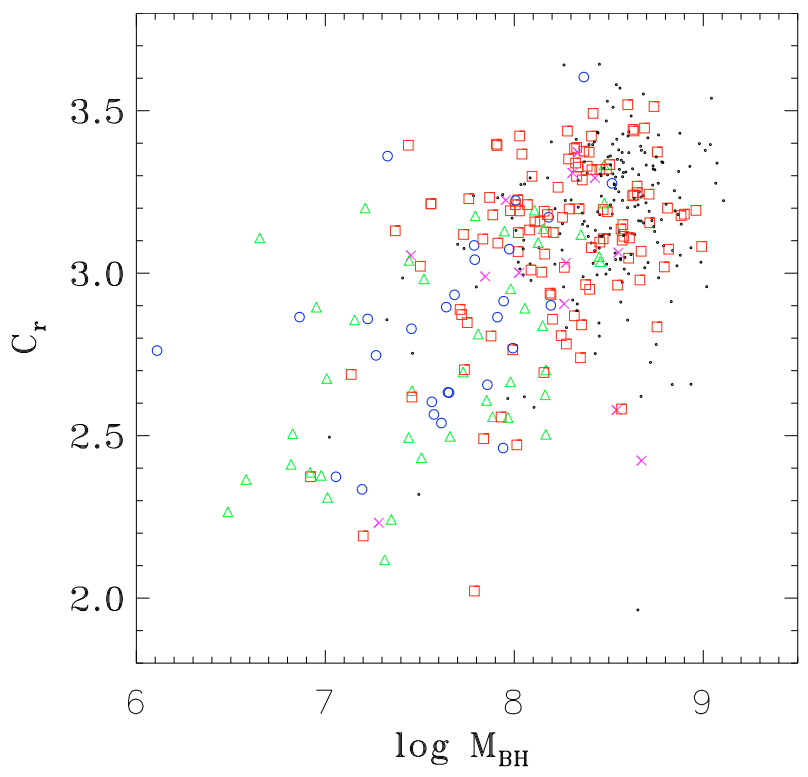

Fig. 5. Logarithm of the black hole mass (in solar units) vs. concentration index, $C_{r}$, for the sub-sample with $0.03<z<0.1$.

the distribution of galaxies in this plane is bimodal, but with a large transition region between $1.4 \lesssim D_{n}(4000) \lesssim 1.7$. Best et al. (2005b) associated the radio emission to an AGN when a galaxy shows an excess $>0.225$ in $D_{n}(4000)$ above the curve corresponding to the prediction of an aging star formation event lasting 3 Gyr and exponentially decaying.

In Fig. 6 we show the location of the SDSS/NVSS sample on $D_{n}(4000)$ versus $L_{1.4 \mathrm{GHz}} / M_{*}$ plane. LEG and UG are mostly clustered well above the threshold curve. Conversely, SFG and HEG occupy a region parallel to the separation curve. The proximity to this curve casts some doubts on the AGN origin of the radio emission in these two classes. Indeed, there is large mis-match in the scales probed by the NVSS image and SDSS spectrum that can cause an erroneous identification of the main mechanism of radio emission. The value of $D_{n}(4000)$ used to derive the age of the stellar population and to predict the radio emission related to star formation is indeed appropriate only for the central $3^{\prime \prime}$ covered by the SDSS fiber. With a substantial gradient in stellar age the predicted value of $L_{1.4 \mathrm{GHz}} / M_{*}$ for the whole galaxy due to star formation could be substantially underestimated. This is case, e.g., for spiral galaxies when the fiber covers only the bulge, while most of the star formation occurs in the spiral arms. We will return to this issue in Sects. 5 and 6.

Finally, we consider the $L_{1.4 \mathrm{GHz}}-L_{[\mathrm{O} \text { III] }}$ plane (Fig. 7), already used in Baldi \& Capetti (2009), now taking advantage of the additional information on the optical classification of the sources. In this plane we note a horizontal stratification of the classes: in order of increasing line luminosity we find SFG and UG, followed by LEG and HEG. Only LEG and UG reach the line-radio correlation defined by the $3 \mathrm{CR}$ sample. Most HEG show a $L_{1.4 \mathrm{GHz}} / L_{[\mathrm{O} \text { III] }}$ ratio much higher than seen in LEG and UG.

Summarizing, the SDSS/NVSS sample is predominantly composed of low excitation AGN hosted by red, quiescent (from the point of view of star formation), ETG and with large $M_{\mathrm{BH}}$. We also include in this main class the spectroscopically unclassified objects that appear to share these features and are most likely simply lower luminosity AGN.

High excitation galaxies are also present but represent only a minority $(\sim 10 \%)$ of the sample, and they usually show ongoing

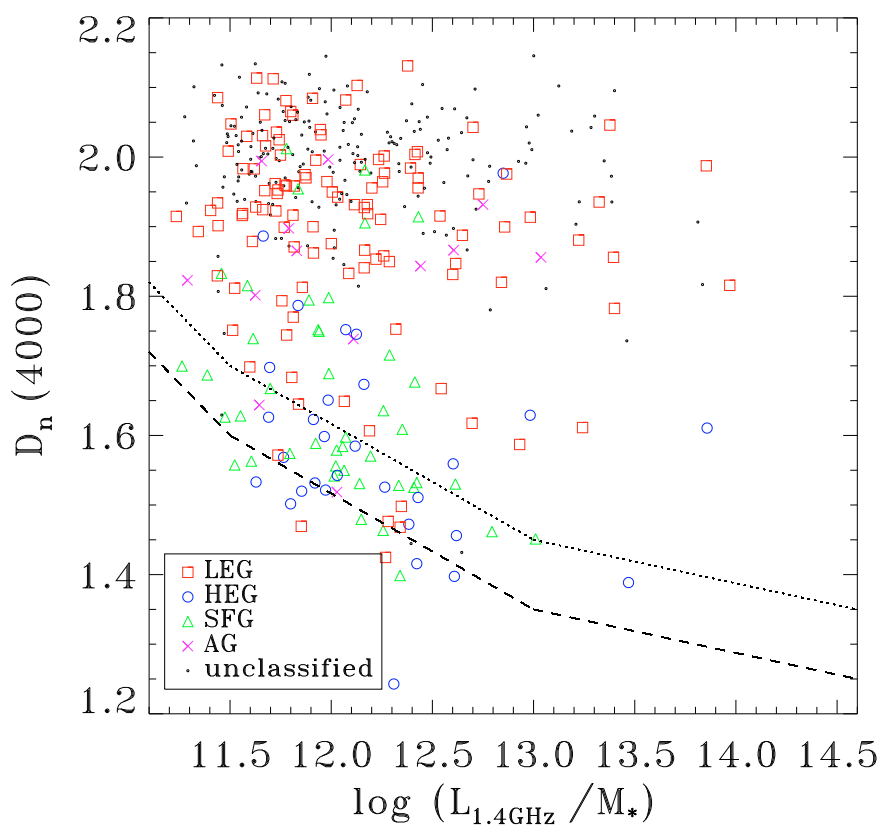

Fig. 6. $D_{n}(4000)$ vs. $L_{1.4 \mathrm{GHz}} / M_{*}$ for the galaxies of SDSS/NVSS sample with $0.03<z<0.1$ and $\sigma\left(C_{r}\right)<0.2$. The dashed curve is the empirical separation between the star forming and AGN radio emitting sources, performed by Best et al. (2005b) (read the text for details). The dotted curve is shifted 0.1 above in $D_{n}(4000)$ of the dashed curve, in order to separate the objects with a possible significant star-formation contribution to their radio emission.

star formation, stronger emission lines and smaller black hole masses than those of LEG, and are often hosted in late-type galaxies. These properties are very similar to those of the starforming galaxies (15\% of the sample), with the very important difference that SFG have a much lower level of [O III] line luminosity (a factor of $\sim 100$ ) than HEG.

\section{Results: the sub-sample with $0.1<z<0.3$}

We now briefly summarize the results obtained from the same analysis described in the previous section, but applied to the objects with higher redshift, i.e. $0.1<z<0.3$. We anticipate that overall the analysis of more distant (and more powerful from the point of view of their line and radio luminosities) SDSS/NVSS sources confirms the main results obtained for the lower redshift objects.

In Fig. A.1 we show the diagnostic spectroscopic diagrams. Not surprisingly the fraction of unclassified galaxies is even more significant than at low redshift, reaching $85 \%$. Indeed, focusing on the 1684 objects with an error on the concentration index smaller than 0.2 , there are $1438 \mathrm{UG}, 130 \mathrm{LEG}, 37 \mathrm{HEG}$, 53 SFG, and 26 AG. The dominance of UG makes it difficult to establish whether the breakdown in the other classes changes with redshift. However, considering the upper limits to the $\mathrm{H} \alpha$ fluxes for UG (see Fig. A.2, upper left panel), similarly to what we found for the near sample, they are substantially offset from HEG, suggesting an identification of UG with a mixture of LEG and SFG. Thus the fraction of HEG appears lower than in the near sample being reduced from $\sim 7 \%$ to $\sim 2 \%$.

In Fig. A.2 (upper right panel) we report the analog of Fig. 3 for the higher redshift objects with the concentration index vs. the $4000 \AA$ break. The bulk of the sample (formed by UG and LEG) is shifted at slightly lower $C_{r}$ and lower $D_{n}(4000)$ as 
R. D. Baldi and A. Capetti: Spectro-photometric properties of the radio-loud AGN population

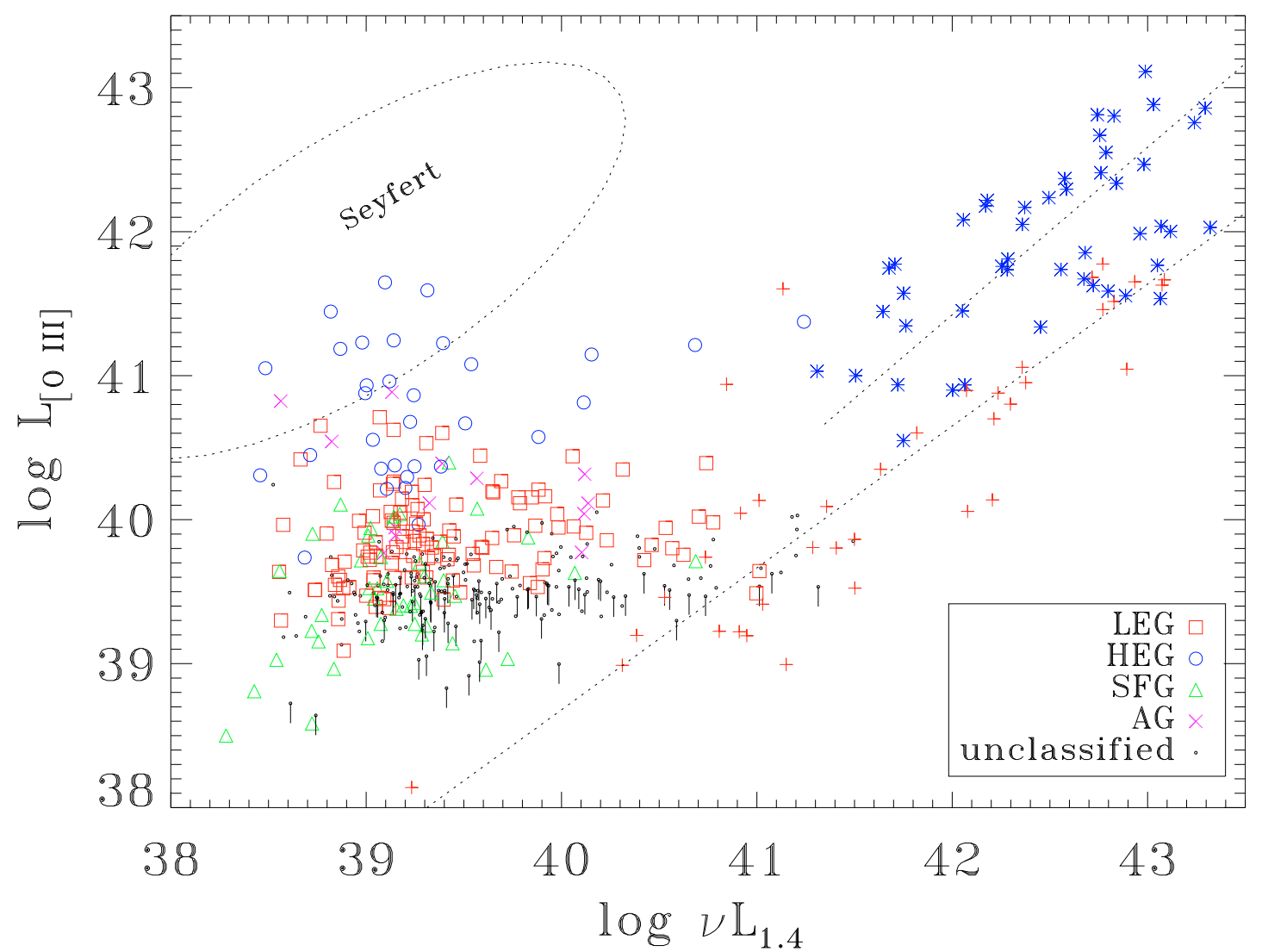

Fig. 7. Logarithm of the radio vs. [O III] luminosities (both in $\mathrm{erg} \mathrm{s}^{-1}$ units) for the $0.03<z<0.1$ sub-sample. The two dashed lines reproduce the line-radio correlation followed by the LEG and HEG of the 3CR sample (red pluses and blue asterisks respectively). The ellipse marks the boundaries of the location of Seyfert galaxies (e.g. Whittle 1985).

expected for the impact of seeing in these more distant objects (Hyde \& Bernardi 2009) and for the younger age of the stellar population. The population with $D_{n}(4000)<1.7$ is still found to be dominated by HEG and SFG, which also form the bulk of the objects with likely late-type morphology.

The location of many of the HEG and SFG in Fig. A.2 (lower left panel), where we compare $D_{n}(4000)$ and $L_{1.4 \mathrm{GHz}} / M_{*}$, is close to the line marking the division between a radio emission associated with an AGN and star formation. However, the fraction of HEG at higher redshift with a clear AGN-origin for the radio emission is larger than that at lower redshift (Fig. 6). Conversely, most LEG and UG at higher $z$ still show an AGN as the dominant process for the production of the radio emission.

In Fig. A.2 we also present (lower right panel) the distributions of the $M_{\mathrm{BH}}$ for each spectroscopic class (the filled portion represents the contribution of galaxies with $C_{r}<2.86$ ). For all classes they are similar to those of the lower $\mathrm{z}$ objects (compare with Fig. 9) with the only exception of SFG with an $M_{\mathrm{BH}}$ larger by a factor of $\sim 10$. Apparently, the dominant population of SFG moves from low mass spiral galaxies, to more massive early-type. Unfortunately we do not have a well defined reference sample of non active ETG for this redshift range to contrast with our results.

Finally, in Fig. A.3 we compare the radio and the [O III] luminosities, similar to what we did in Fig. 7. The selection of more distant objects causes a general shift toward higher luminosities on both axes, but the stratified position of the various classes is confirmed.

\section{Comparison with $3 \mathrm{CR}$ radio-galaxies}

In this section we will draw a quantitative comparison between the spectro-photometric properties of the SDSS/NVSS sources with those of the powerful radio-galaxies part of the 3CR sample.

\subsection{Low excitation galaxies}

Because from the point of view of the optical spectra, most of the SDSS/NVSS radio sources are classified as low excitation galaxies, we start with a comparison with the LEG of 3CR sample ${ }^{6}$.

We here compare the radio morphology of the two samples. We visually inspected all UG and LEG at $z<0.1$, finding that their majority of them is unresolved or barely resolved at the $5^{\prime \prime}$ FIRST resolution, corresponding to a limit to their size of $\$ 10 \mathrm{kpc}$. Nonetheless we found $6(5 \%)$ LEG and 58 (27\%) UG with well resolved radio structures, all of these with $v L_{r} \gtrsim 2 \times 10^{39} \mathrm{erg} \mathrm{s}^{-1}$. The fraction of extended radio sources increases with radio luminosity and, at the higher radio powers, most of them are well resolved (see Fig. 8). All morphological classes are represented, including twin-jets and core-jet FR I, Narrow and Wide Angle Tails, and FR II with clear hot spots. These classes are all found among the 3CR/LEG which, however, are all well resolved with physical sizes ranging from 15 to

${ }^{6}$ We consider as "LEG" sources all those classified as low excitation galaxies, see Buttiglione et al. (2010), from the optical spectra independently of their radio morphology. 

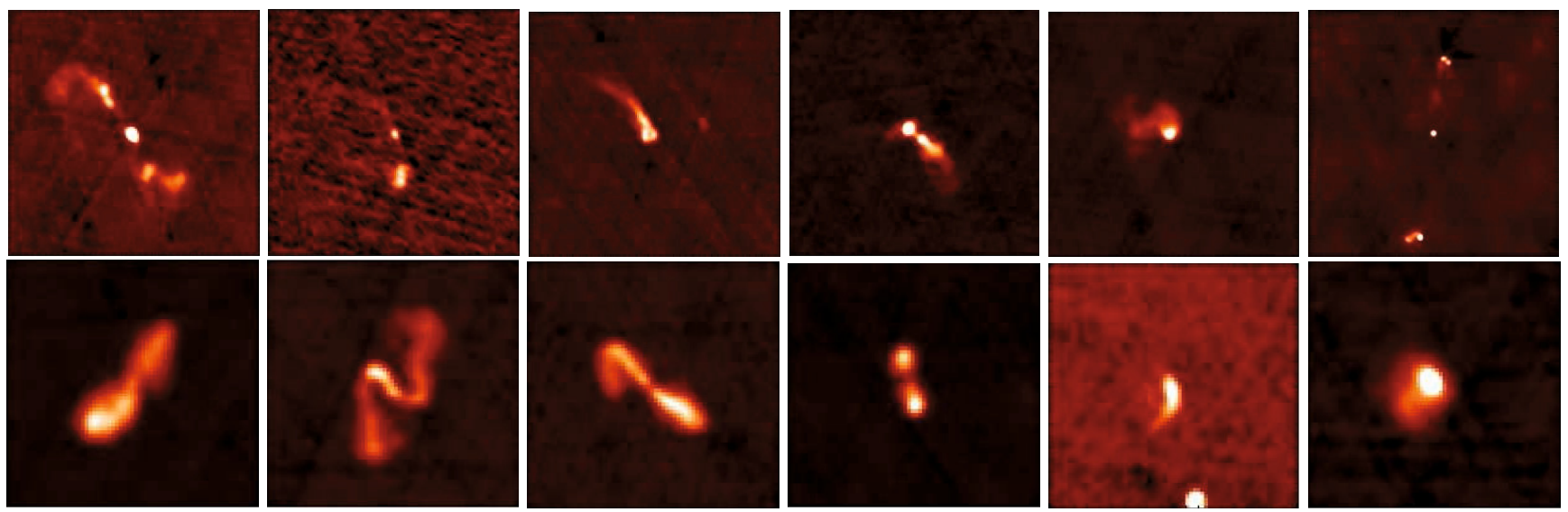

Fig. 8. FIRST images of the 12 brightest SDSS/NVSS radio sources with $z<0.1$ associated with UG and LEG, with a [O III] detection. The fields of view are $4^{\prime} \times 4^{\prime}$ and $2^{\prime} \times 2^{\prime}$ for the top and bottom row respectively. The physical sizes range from 45 to $400 \mathrm{kpc}$.

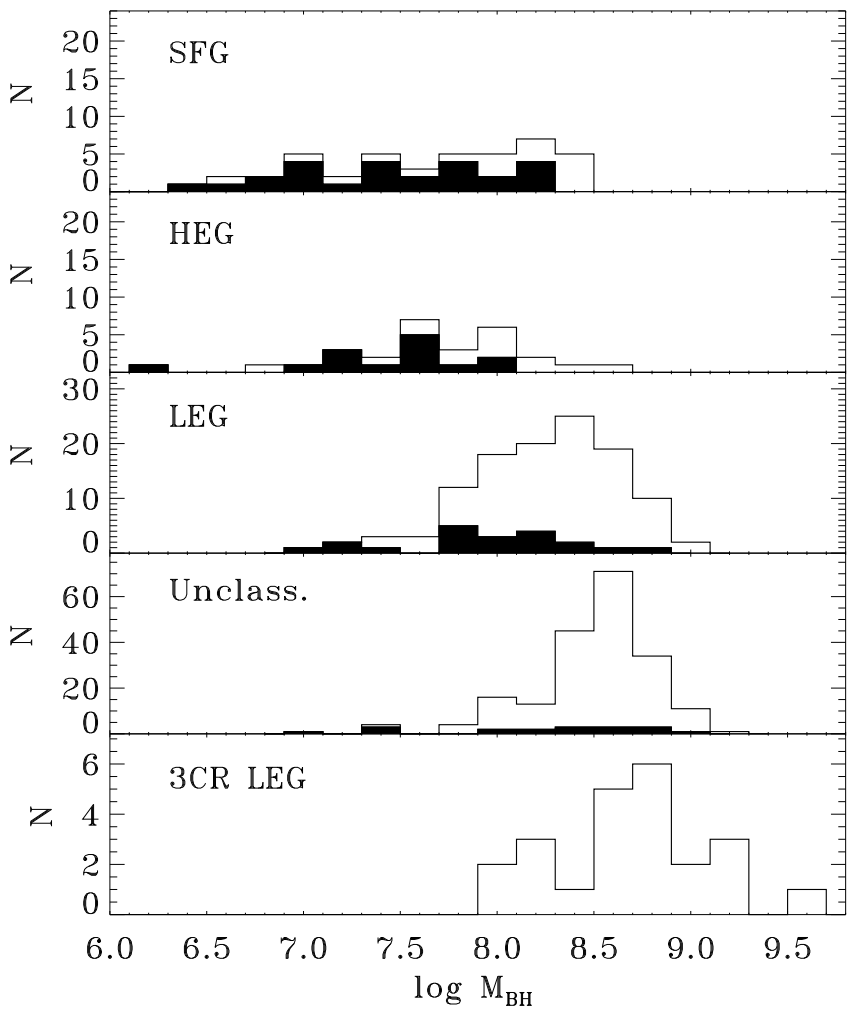

Fig. 9. Distributions of the SMBH mass (in solar units) for the various spectroscopic SDSS/NVSS sub-samples compared to that of LEG part of the 3CR sample. The filled portion of the histograms represents the contribution of galaxies with $C_{r}<2.86$.

$400 \mathrm{kpc}$, in the same range of the SDSS/NVSS sources shown in Fig. 8.

In Fig. 9 we analyze the SMBH mass distribution of each spectroscopic class in comparison with that of $3 \mathrm{CR} / \mathrm{LEG}$. These powerful radio sources have a black hole mass in the range $\left(8.0<\log \left(M_{\mathrm{BH}} / M_{\odot}\right)<9.5\right)$ similar to those of the LEG and UG of the SDSS/NVSS sample. Indeed, the vast majority of these objects have a black hole mass in the range $7.7<$ $\log \left(M_{\mathrm{BH}} / M_{\odot}\right)<9.5$. A low mass tail, with $\log \left(M_{\mathrm{BH}} / M_{\odot}\right)<$ 7.5 , is present among LEG and UG but amounting to only $3 \%$ of the galaxies and mostly composed of late-type galaxies (these sources will be discussed in more detail in Sect. 6). If we consider only the ETG, the median logarithm of $M_{\mathrm{BH}}$

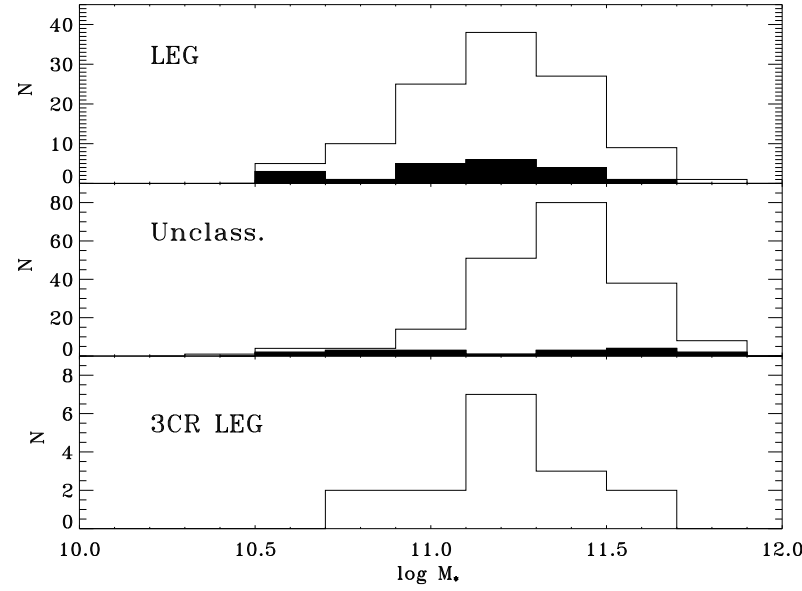

Fig. 10. Distributions of the stellar masses (in solar units) for the samples of LEG and unclassified galaxies in the SDSS/NVSS sample, compared to that of LEG part of the 3CR sample with $z<0.1$. The filled portion of the histograms represent the contribution of galaxies with $C_{r}<2.86$.

in solar units are 8.30, 8.52, and 8.44 for LEG, UG and the combined "LEG+UG" sample respectively. $M_{\mathrm{BH}}$ in $3 \mathrm{CR} / \mathrm{LEG}$ are only slightly larger than in UG and LEG, with a median of $\log M_{\mathrm{BH}} / M_{\odot}=8.67$. A Kolmogorov-Smirnov test indicates that the distributions of SMBH for UG+LEG and 3CR/LEG are not statistically different at a significance limit of $99 \%$. As already noted, SFG and HEG have instead lower $M_{\mathrm{BH}}$ and a large contribution of late type galaxies.

In Fig. 10 we show the distribution of the host masses, estimated from the $g$ and $z$ SDSS magnitudes (Bell et al. 2007) restricting to LEG and UG sources, and of the 17 3CR/LEG with available SDSS images. They share the same host mass range $10.5<\log \left(M_{*} / M_{\odot}\right)<12$. The median host masses are $\log$ $\left(M_{*} / M_{\odot}\right)=11.18,11.37$ and 11.26 for LEG, UG and 3CR/LEG respectively. Using a Kolmogorov-Smirnov test, we found that these small differences are not statistically significant.

Considering now the morphology of the host galaxies, 3CR/LEG are found in elliptical galaxies (e.g. Martel et al. 1999; Madrid et al. 2006) similarly to the typical hosts of SDSS/NVSS galaxies.

From the point of view of their broadband color, we consider the location of the 3CR/LEG in the $M_{r}$ vs. $u-r$ plane 
(see Fig. 4), looking for blue galaxies. All the 3CR/LEG sources are red, with the exception of 3C 236 and 3C 084 . The color of the latter object is however dominated by the bright, highly polarized, and variable optical nucleus (Martin et al. 1976), typical of BL Lac objects. Instead 3C 236 shows UV knots related to star formation (O'Dea et al. 2001), indicating only one blue galaxy among the $163 \mathrm{C} / \mathrm{LEG}$. This fully agrees with the finding of Baldi \& Capetti (2008) based on the analysis of their near UV HST images.

Summarizing, the hosts of 3CR/LEG and those of LEG in the SDSS/NVSS sample are typically red quiescent early-type galaxies, with distributions of black holes and stellar masses that are statistically indistinguishable. The most striking difference between the two samples is related to the radio morphology, i.e. to the large fraction of objects $(\sim 80 \%)$ in the SDSS/NVSS that, despite having a line luminosity matched to the 3CR/LEG, do not show extended radio structures.

\subsection{High excitation galaxies}

About half of the 3CR sources with $z<0.3$ are HEG (Buttiglione et al. 2010). They are all bright radio-sources (log $v L_{178}\left[\mathrm{erg} \mathrm{s}^{-1}\right] \gtrsim 41$ ) with a FR II morphology. About $1 / 3$ of them show broad emission lines. Their distribution of SMBH masses covers the range $\log M_{\mathrm{BH}} / M_{\odot}=8.1-9.5$ with a median of $8.75^{7}$.

In the SDSS/NVSS sample the HEG are instead a minority, $7 \%$ in the sub-sample of nearby objects $(0.03<z<0.1)$ and only $2 \%$ in the "far sample" with $(0.1<z<0.3)$ for a total of 67 objects $^{8}$.

Considering their radio morphology, only a minority $(\sim 20 \%)$ have resolved structures in FIRST, and only seven of them are clear FR II. These are all high-power sources $v L_{r} \gtrsim 10^{41} \mathrm{erg} \mathrm{s}^{-1}$, a low limit similar to that found for $3 \mathrm{CR} / \mathrm{HEG}$.

HEG in the SDSS/NVSS sample are generally blue ( $60 \%)$ and with a strong level of star formation (25 out of 30 have $\left.D_{n}(4000)<1.7\right)$, which is in line with $3 \mathrm{CR} / \mathrm{HEG}$ (Baldi \& Capetti 2008; Smolčić 2009). But SDSS/NVSS HEG are hosted almost in the same fraction by early and late-type galaxies, which is at odds with the elliptical morphology of 3CR/HEG hosts. Furthermore, their SMBH masses distribution is shifted by about one order of magnitude (the median is $\log M_{\mathrm{BH}} / M_{\odot}=$ 7.88) toward smaller masses compared to $3 \mathrm{CR} / \mathrm{HEG}$. In addition, in the $D_{n}(4000)$ vs. $L_{1.4 \mathrm{GHz}} / M_{*}$ plane (Fig. 6) two thirds of HEG are located close (with an offset $<0.1$ in $D_{n}(4000)$ ) to the boundary of the region characteristic of the objects in which the radio emission is powered by star formation. Although these are certainly AGN, given their emission line ratios, they might enter the SDSS/NVSS sample only because of a high level of star formation that powers their radio emission. This should be taken into account when dealing with AGN radio luminosity functions derived from this sample.

Let us focus on the location of SDSS/NVSS HEG (including all objects with $0.03<z<0.3$ ) in the $L_{1.4 \mathrm{GHz}}$ vs. $L_{[\mathrm{O} \text { III] }}$ plane

\footnotetext{
7 The black hole masses for 3CR/HEG have been estimated from their $\mathrm{H}$-band absolute magnitude (after removal of the nuclear contribution estimated by Baldi et al. 2010) as tabulated by Buttiglione et al. (2010), converted into $K$-band luminosity assuming $H-K=0.21$ (Mannucci et al. 2001), and using the $L_{K}-M_{\mathrm{BH}}$ relation (Marconi \& Hunt 2003).

8 All these objects only show narrow emission lines. Indeed Best et al. (2005b) excluded the objects classified as QSO (that instead generally do show broad lines) by the automated SDSS classification pipeline, because a bright nucleus would have prevented a detailed study of the host properties.
}

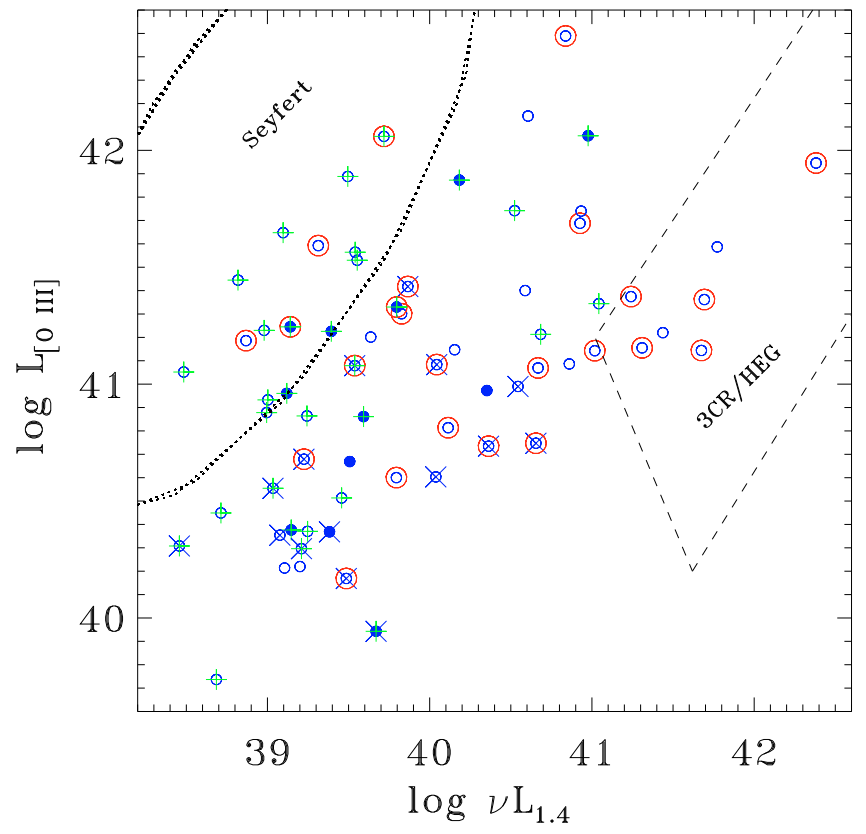

Fig. 11. Logarithm of the radio vs. [O III] luminosities for the HEG with $0.03<z<0.3$. The symbols added to the empty circles correspond to: 1) filled points are late-type galaxies; 2) large red circles have black hole masses $\left.\log M_{\mathrm{BH}} / M_{\odot}>8 ; 3\right)$ the green plusses are the objects close to the empirical separation between the star forming and AGN radio-emitting sources; 4) the blue crosses are the objects that would be classified as LEG adopting the HEG/LEG separation derived for 3CR galaxies by Buttiglione et al. (2010). We draw as a comparison the location of other classes of high excitation AGN: the ellipse marks the boundary of the region characteristic of Seyfert (Whittle 1985), while the dashed region comprises the 3CR/HEG (Buttiglione et al. 2010).

(Fig. 11). They form the high end of the luminosity distribution in emission lines $\left(L_{[\mathrm{O} I I I]} \gtrsim 10^{40} \mathrm{erg} \mathrm{s}^{-1}\right)$ and their radio total luminosities span a large range (from $10^{38.5}$ to $10^{42.5} \mathrm{erg} \mathrm{s}^{-1}$ ). At high emission line luminosities $\left(L_{[\mathrm{O} \text { III] }} \gtrsim 10^{41} \mathrm{erg} \mathrm{s}^{-1}\right)$ a few objects are located in the region characteristic of $3 \mathrm{CR} / \mathrm{HEG}$, but HEG also reach the locus of Seyfert galaxies (Whittle 1985), without any apparent discontinuity. Moving from high to low radio luminosities, there is a trend in the host properties: high $L_{1.4 \mathrm{GHz}}$ sources are exclusively ETG (empty circles), while those of lower radio luminosity, falling in the Seyfert region, are still mostly ETG but with a contribution from late-types (filled circles) and with many objects with a likely possibility of a star-formation origin for their radio emission ("+" symbols). The black hole mass (large red circles mark galaxies with log $\left.M_{\mathrm{BH}} / M_{\odot}>8\right)$ does not seem to have any simple relation with the location of the objects in the $L_{1.4 \mathrm{GHz}}-L_{[\mathrm{O} \text { III] }}$ plane.

At lower line luminosities, the situation is somewhat different. In the 3CR there are no HEG below a line luminosity of $10^{41} \mathrm{erg} \mathrm{s}^{-1}$ and similarly there are no SDSS/NVSS HEG extending the correlation between line and radio luminosities defined by 3CR HEG toward lower powers. Among the objects with $L_{[\mathrm{OIII}]} \lesssim 10^{41} \mathrm{erg} \mathrm{s}^{-1}$ there is also a substantial fraction of sources that would be classified as LEG by adopting the HEG/LEG classification of RLAGN (crossed circles). There are also many low mass, late-type galaxies.

Thus SDSS/NVSS HEG appear to be rather different from those found in the 3CR sample, with only a few of them with properties consistent to those of these powerful FR II radiogalaxies. SDSS/NVSS HEG are instead more often associated with smaller mass and later type galaxies, similar to the host of 
Seyfert galaxies with which they also share the location in the line vs. radio luminosity plane. The combination of these characteristics suggests that the SDSS/NVSS HEG sub-sample contains a large fraction of sources that would classically be considered as radio-quiet AGN and a minority of RLAGN.

Interestingly, several HEG lie in between Seyfert and $3 \mathrm{CR} / \mathrm{HEG}$ (i.e., between radio-quiet and radio-loud AGN) without a clear discontinuity in either the host or the AGN properties.

\subsection{Star-forming galaxies}

In the $3 \mathrm{CR}$ sample there is only one galaxy classified as $\mathrm{SFG}^{9}$. The fraction SFG in the SDSS/NVSS sample is substantially higher, $\sim 11 \%$ in the low $-z$ sample, although we remind the reader that the method used to classify the SFG may lead to an overestimate of the number of these objects. This difference may be related to the higher AGN power of the $3 \mathrm{CR}$ sources that impedes observing the effects of star formation in their optical spectra. The a priori exclusion of SFG could also bias our estimate of the fraction of "blue" ETG derived in Sect. 3.2.

However, the properties of SFG are in general incompatible with the interpretation that they are simply the result of a combination of a radio loud LEG nucleus with a higher level of star formation. Indeed, they usually have lower $M_{\mathrm{BH}}$ than LEG/UG and are mostly found in late-type galaxies. Furthermore most SFG are close to the curve that separates objects in which the radio emission is powered by an AGN and by star-formation (see Fig. 6) and are generally well offset from LEG/UG, which argues for a substantial contamination of their radio luminosity from non nuclear processes.

Only a minority of SFG ( $\sim 5-10$ objects, depending on the diagram considered) have spectro-photometric properties (leaving aside the emission line ratios) similar to those of LEG/UG. These could indeed be LEG/UG from the nuclear point of view, but with a higher star formation rate. Six SFG in particular are hosted in ETG with a blue color (see Fig. 4). Including them among the blue galaxies would raise their overall fraction to $7 \%$, still consistent with the the fraction of star formation in quiescent ETG (Schawinski et al. 2009).

Conversely, SFG can in part be the result of the combination of a HEG nucleus with a high level of star formation. Indeed, the properties of the hosts of the two classes are similar. A varying relative contribution of the active nucleus and of star formation to the emission lines can account for the broad range of diagnostic line ratios covered by SFG and HEG.

\section{Are there exceptions to the rule?}

The SDSS/NVSS sources are thus composed mainly of objects with low excitation spectra and their host galaxies are mostly red $^{10}$, massive early-type galaxies, harboring SMBH of very large masses. These characteristics are shared by the LEG drawn from the 3CR sample.

As shown in the previous sections, there is a minority of radio sources associated with galaxies with an HEG or SFG emission line spectrum, which are also found in smaller hosts, often

\footnotetext{
9 This is 3C 198, a bright FR II, hosted by a blue elliptical galaxy $\left(C_{r}=3.1\right)$, with $D_{n}(4000)=1.1$, and with $\log M_{\mathrm{BH}} / M_{\odot}=8.2$.

10 As discussed before, this is the general rule for the hosts of low excitation RLAGN, and the fraction of objects with signs of star formation are very similar in the $3 \mathrm{CR}$ and SDSS/NVSS samples and in quiescent (non AGN) ETG.
}

of late type. However, these sub-classes suffer from a strong contamination from radio-quiet AGN or from objects whose radio emission is powered by star formation.

At this stage it is important to establish whether there are exceptions to these general rules answering to the questions: can a RLAGN be associated with a small black hole? Are there RLAGN hosted in spiral galaxies? We will focus on the "near sample" $(0.03<z<0.1)$, for which we have better spectrophotometric information and, which is still sufficiently large to contain relatively rare outliers, when compared to, e.g., the 34 $3 \mathrm{CR}$ in the same redshift range.

The black hole mass distribution of the 315 LEG (or UG) has a short tail of low $M_{\mathrm{BH}}$, while in the 3CR there is sharp cut-off in the black hole mass distribution at $\log \left(M_{\mathrm{BH}} / M_{\odot}\right) \sim 7.7$. We will then focus on the 11 SDSS/NVSS objects with $\log \left(M_{\mathrm{BH}} / M_{\odot}\right)<$ 7.5. There are also $11 \mathrm{LEG} / \mathrm{UG}$ with $C_{r}<2.6$, thus likely latetype galaxies (four of them also have $\log \left(M_{\mathrm{BH}} / M_{\odot}\right)<7.5$ and are included in the sample of galaxies of low SMBH mass). Furthermore, spiral hosts could also be found among the objects with $C_{r}>2.86$, because $\sim 1 / 3$ of $\mathrm{Sa}$ and $13 \%$ of Sb galaxies make this selection (see Bernardi et al. 2010).

Before we can conclude that any of these are genuine exceptions we examine their properties in closer detail. We performed an object by object inspection of the position in spectrophotometric diagnostic diagrams, and of their optical and radio images.

First of all we looked for spirals in the sub-sample with $C_{r}>2.86$. Bernardi et al. (2010) showed that late and earlytype galaxies have different distributions of axial ratios, but with a substantial overlap. Thence ellipticity cannot be used to assess the possible contribution of spirals to the RLAGN population. To explore this we are left only with direct visual inspection of the SDSS images. We then considered the 277 galaxies with $z<0.1$ having $C_{r}>2.86, \sigma\left(C_{r}\right)<0.2$, and a UG or LEG classification. Although we extended this method to a higher redshift than used by e.g. the Zoo Project, we are confident that the quality of the SDSS images is sufficient for a robust identification of their Hubble type. This conclusion can be drawn looking at the images in Fig. 12, which shows 20 galaxies selected at random from those in the highest redshift bin considered, $0.09<z<0.1$, all of them clearly ETG. The high reliability of visual inspection at these relative high redshift probably arises because we are dealing in most cases with very massive objects, whose morphology remains well defined even at $z \sim 0.1$.

We only found four galaxies whose type cannot be defined securely because they are seen almost edge-on (see the bottom row in Fig. 12) and which, in principle, could be spirals. However, if indeed massive late-type galaxies were associated with RLAGN, we should also have found in our analysis their face-on counterparts. This leads us to conclude that the four galaxies under scrutiny are most likely the small fraction of S0 expected to be seen edge-on.

We then consider the 11 galaxies with $C_{r}<2.6$. The first issue is to establish whether their radio emission is indeed powered by an AGN and not by star formation. As shown in Fig. 6, nine LEG/UG are located close (with an offset of $<0.1$ in $\left.D_{n}(4000)\right)$ to the boundary of the region characteristic of the objects in which the radio emission is powered by star formation; six of them are galaxies with $C_{r}<2.6$. As discussed in Sect. 3.2 this casts doubts on the AGN origin of the radio emission in these objects.

This leaves us with only five galaxies with $C_{r}<2.6$ with radio emission of apparently secure AGN origin. Visual inspection of their SDSS images clearly indicates that four of them $(\sim \% 1$ 
R. D. Baldi and A. Capetti: Spectro-photometric properties of the radio-loud AGN population

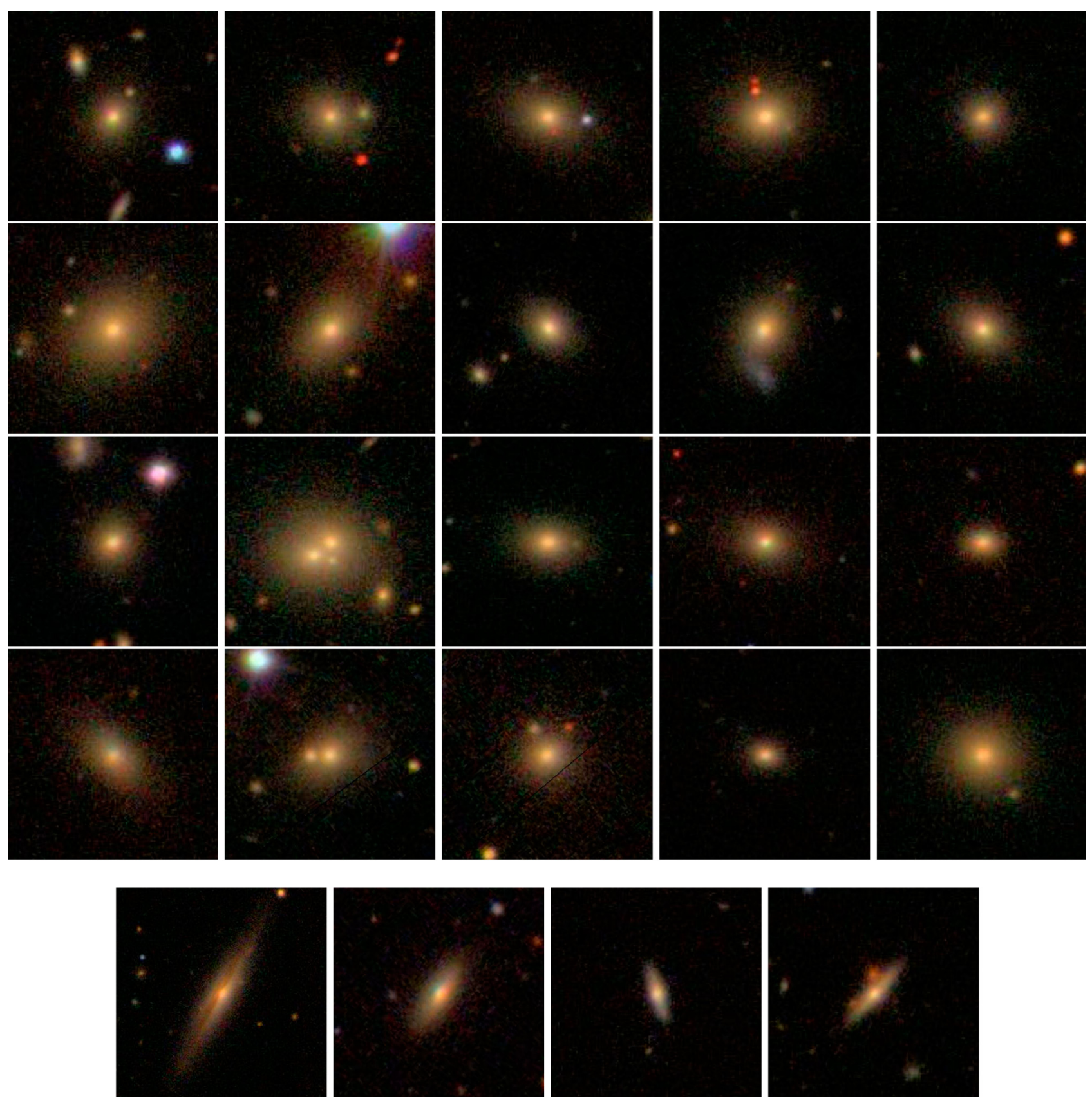

Fig. 12. First four rows: $50^{\prime \prime} \times 50^{\prime \prime}$ SDSS images of 20 randomly selected LEG or UG in the redshift bin $0.09<z<0.1$. All of them can be classified by visual inspection as ETG. Bottom row: four galaxies whose Hubble type cannot be defined because they are seen edge-on.

of the sample) are clearly ETG. This is not surprising because Bernardi et al. (2010) estimated that the fraction of galaxies visually classified as ETG with $C_{r}<2.6$ is $\sim 2 \%$ for Es and $\sim 7 \%$ for S0s. Limited to the most massive ones $\left(M_{r} \lesssim-21\right)$ these fractions are approximately halved. Only one galaxy is indeed a spiral (SDSS J170007.17+375022.2) at a redshift of 0.063 . However, the $3^{\prime \prime}$ SDSS fiber covers only its bulge, returning a $D_{n}(4000)=1.75$, typical of a rather old stellar population. This is the value used to locate this source in the $D_{n}(4000)$ vs. $L_{1.4 \mathrm{GHz}} / M_{*}$ plane. We argue that a substantial fraction of its radio emission can originate from younger stars within the spiral arms, an idea supported by the lack of a central source in the FIRST image.

We finally consider the seven galaxies with low $M_{\mathrm{BH}}$ (four were already discussed along with the likely late type). Although the radio emission of one of them is probably dominated by star formation, another three possibly make the $5 \mathrm{mJy}$ threshold only thanks to the contribution of unrelated nearby radio sources, there are three galaxies behaving like the rest of the LEG/UG population is all diagnostic diagrams, but with $M_{\mathrm{BH}} \sim$ $2-3 \times 10^{7} M_{\odot}$. One of them (SDSS J133453.37-013238.5) is associated with a radio source of clear FR II morphology extended over $\sim 90 \mathrm{kpc}$, an unmistakable sign of the presence of a RLAGN.

Nonetheless, the quoted SMBH masses are not actual measurements, but estimates derived from the stellar velocity dispersion, $\sigma_{*}$. Because this relation has an intrinsic scatter, a tail of low $\sigma_{*}$ is indeed expected in a population with a low-mass cut-off in black hole masses. Without a prior knowledge of the SMBH masses distribution it is difficult to estimate whether our 

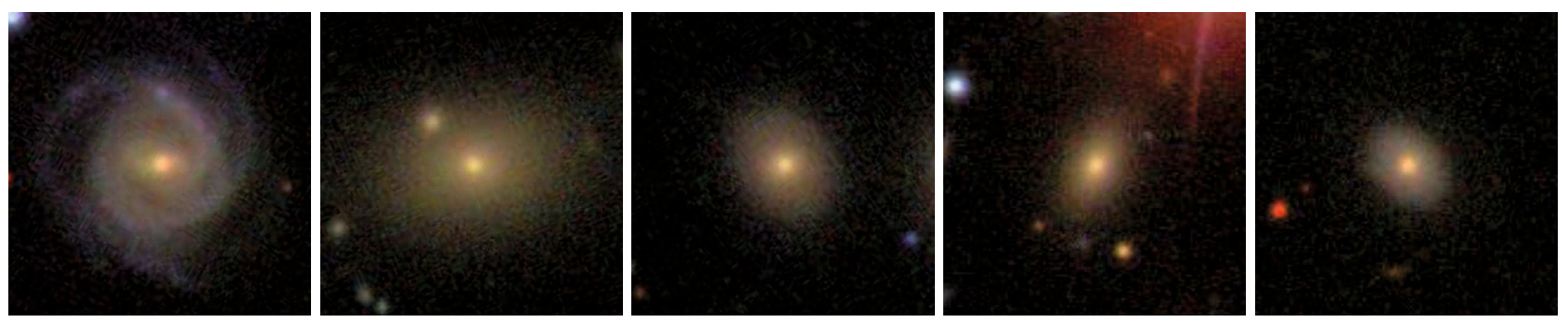

Fig. 13. $50^{\prime \prime} \times 50^{\prime \prime}$ SDSS images of the five putative late-type galaxies, with $C_{r}<2.6$, all but the one on the left are clearly ETG.

fraction of low $\sigma_{*}$ objects $(\sim 1 \%$ of the sample) is consistent with this interpretation. We then used the relation between the host and the SMBH masses derived by Marconi \& Hunt (2003) to obtain an independent $M_{\mathrm{BH}}$ measurement. Their host masses are in the range $\log M_{*} / M_{\odot}=10.54-10.99$ corresponding to $\log \left(M_{\mathrm{BH}} / M_{\odot}\right) \sim 7.93-8.37$.

Summarizing, we failed to find any convincing RLAGN associated with a spiral galaxy or with black holes with a mass substantially smaller than to $10^{8} M_{\odot}$.

However, a link between $M_{\mathrm{BH}}$ and radio luminosity exists. Indeed, the normalization of the radio luminosity function of galaxies of different SMBH masses shows a dependence as $\rho \sim M_{\mathrm{BH}}^{1.6}$ (Best et al. 2005a). Thus AGN associated with smaller $\mathrm{SMBH}$ are expected to be less frequent at a given threshold in radio luminosity than in $\mathrm{SMBH}$ of higher mass. The stellar mass function of ETG is essentially constant (in a log-log representation) for $M_{*} / M_{\odot} \lesssim 10^{11}$ (e.g. Bernardi et al. 2010), corresponding to $M_{\mathrm{BH}} / M_{\odot} \sim 2 \times 10^{8}$, after adopting the ratio $M_{\mathrm{BH}} / M_{*}$ from Marconi \& Hunt (2003). The number of LEG/UG radiogalaxies with $7.7 \lesssim \log M_{\mathrm{BH}} / M_{*} \lesssim 8.7$ is $\sim 300$ and the number of expected radio-galaxies in the SDSS/NVSS sample with $6.7 \lesssim \log M_{\mathrm{BH}} / M_{*} \lesssim 7.7$ is $\sim 300 \times 10^{-1.6} \sim 7$. The failure to find any of these low-mass objects indicates that the link between radio luminosity and $M_{\mathrm{BH}}$ breaks toward low $\mathrm{SMBH}$ masses.

\section{Summary and conclusions}

In a previous paper (Baldi \& Capetti 2009) we showed that the sources selected by Best et al. (2005b) by cross-correlating the NVSS, FIRST, and SDSS surveys (the SDSS/NVSS sample) do not follow the correlations between line and radio luminosity defined by the radio-galaxies part of samples with a higher flux threshold, such as the $3 \mathrm{CR}$, the B2, and the $2 \mathrm{Jy}$. They are characterized by a large deficit of radio emission at a given level of emission line luminosity with respect to these more powerful radio-sources. We argued that the prevalence of sources with luminous extended radio structures in high-flux limited samples is due to a selection bias. The SDSS/NVSS sources form the bulk of the local radio-loud AGN population, but they are still poorly explored.

We here analyze their spectro-photometric properties looking for differences in their hosts and in their optical spectra, which might explain their different radio properties. The majority of the SDSS/NVSS sources have emission line ratios characteristic of low excitation galaxies. A comparison with the LEG part of the 3CR sample show that the SDSS/NVSS hosts are indistinguishable from their counterparts of higher radio power from the point of view of their morphology, distribution of black hole and stellar masses, and broad band colors. Indeed the hosts of both samples are massive, red, early-type galaxies, with black hole masses in the range $7.7 \lessgtr \log \left(M_{\mathrm{BH}} / M_{\odot}\right) \lesssim 9.5$. A small percentage $(\sim 5 \%)$ of objects with signs of recent star formation is also present in both samples, in a similar fraction with respect to the population of quiescent (from the point of view of nuclear activity) galaxies. Thus the differences in radio power between SDSS/NVSS and 3CR sources (matched at a given level of emission line luminosity) cannot be ascribed to their hosts being different.

Environment can also play a role. Indeed, the confinement of the radio emitting plasma produced by the hot intra-group or intra-cluster medium is more effective in a dense environment than in regions of lower galaxies densities. In this latter case, the rapid expansion of the relativistic plasma causes dramatic adiabatic losses, lowering the observed radio power. However, Best et al. (2005b) show that SDSS/NVSS are already in general found in richer environments than normal galaxies. A detailed comparison of the environment of SDSS/NVSS and 3CR sources is needed to address this issue, which be done in a forthcoming paper.

In Baldi \& Capetti (2009) we argued that SDSS/NVSS sources could be relatively young and their extended radio emission has yet to fully form. This requires that the radio luminosity increases with time as the source expands, slowly burrowing its way into the interstellar medium of the host galaxy. The results presented here provide us with a further piece of evidence in favor of an evolutionary sequence in radio-loud AGN. Indeed we found that unclassified galaxies are more often associated with extended radio sources with respect to low excitation galaxies (the percentages are $27 \%$ and $5 \%$ for UG and LEG, respectively). This is counter-intuitive, because LEG are brighter in line emission than UG; one might have expected a higher line luminosity, generally indicative of a higher AGN power, to correspond more often to a well developed radio structure. However, the line luminosity also evolves with time and with the size of the radio source. The compression of the ambient medium due to the passage of the jet head (and to the lateral expansion shocks) increases the density of the line-emitting clouds and consequently (given the dependence of line luminosity on the density squared) their line luminosity. The line luminosity increases with time, because the jets compress more clouds (see e.g. Labiano 2008) until the radio source is confined to within the host galaxy. Even larger sources propagate in regions of lower gas densities. At a given level of the ionizing continuum, they are expected to be less luminous in line that smaller sources, where the jet-cloud interactions are more efficient. Thus a radio-galaxy moves with time in the $L_{1.4 \mathrm{GHz}}-L_{[\mathrm{O} \text { III] }}$ plane along a complex evolutionary track. The higher fraction of UG with extended radio structures can be due to the evolution of part of the LEG population from small radio sources of high $L_{[\mathrm{O} \text { III] }}$ to larger size and smaller $L_{[\mathrm{O} \text { III] }}$.

Additional indicators of the jet and AGN power are needed to further compare high and low radio power sources. These can be obtained, for example, from measurements of the radio cores (from images of higher resolution and at higher frequency than 
the FIRST maps currently available) or of the higher frequency nuclear emission by using, e.g., HST or Chandra imaging. In particular it is crucial to test whether also the sources belonging to the SDSS/NVSS sample obey the relation between radio core and line emission spanning from miniature to high-power radio-galaxies (Baldi \& Capetti 2009). This would be a clear indication that there is indeed a close connection between the radiative and kinetic output in radio-loud AGN, but that the best indicator of jet power is the radio core luminosity and not the total radio emission. This interpretation is in line with the proportionality of core and jet power derived from the estimates of the energy required to form the cavities observed in X-ray images (Heinz et al. 2007). Better radio images will also provide us with a measurement of the core dominance, to test the prediction that the deficit of radio power of SDSS/NVSS sources is indeed related to a higher core dominance with respect to, e.g., $3 \mathrm{CR}$ objects, in analogous to the results we obtained for miniature radio-galaxies.

After a detailed analysis on a object by object basis, we concluded that no bona-fide radio-loud AGN with low excitation spectra could be found in a late-type host or with black holes with a mass substantially smaller than $10^{8} M_{\odot}$. The SMBH mass distributions of 3CR/LEG and in the SDSS/NVSS samples suggests that a RLAGN LEG can only be associated with relatively large mass of the central object. This might represent a very valuable information in our quest for the origin and evolution of RLAGN (see e.g. Chiaberge 2008) and of their host galaxies. Nonetheless, because there is a link between $M_{\mathrm{BH}}$ and the normalization of the radio luminosity function, RLAGN might be associated with low SMBH masses and not be represented in the sample because they are too rare. However, we estimated that seven low $M_{\mathrm{BH}}$ objects would be expected, while none was found. This idea can be tested to a greater extent by exploring the radio properties of smaller mass ETG, harboring black holes around $10^{7} M_{\odot}$.

A minority $(\sim 10 \%)$ of the SDSS/NVSS sample shows rather different properties, with a spectrum typical of high excitation or star-forming galaxies. They strikingly differ from LEG: they have a wider distribution of SMBH masses, ranging from $\sim 10^{6} M_{\odot}$ to $\sim 10^{9} M_{\odot}$ and are often found in spiral galaxies. Only a few of the HEG in the SDSS/NVSS sample (less than $\sim 10$ objects) have spectro-photometric properties similar to the HEG found in the 3CR. Furthermore, there might be a contamination from radio-quiet galaxies with high excitation spectra, whose radio emission is dominated by star formation.

It is interesting that there is no clear discontinuity in either the host or the AGN properties in the SDSS/NVSS sample moving from galaxies that lie in the region characteristic of Seyfert to that covered by $3 \mathrm{CR} / \mathrm{HEG}$, objects that classically would be defined as radio-quiet and radio-loud AGN. This issue certainly deserves further studies, which requires collecting additional multiband data of these galaxies.

It is possible that the same situation occurs also for LEG. The density of SDSS/NVSS sources increases at given line luminosity toward lower radio luminosity (with a dependence well described by a power law $N \sim L_{1.4 \mathrm{GHz}}^{-1}$ ) down to the completeness limit imposed by the radio flux limit. We cannot establish whether a discontinuity exists between the RLAGN discussed here and the population of radio-quiet LEG, i.e. the LINER. An extension of this study toward even lower radio flux limits, probably at sub-mJy level, is required to show a possible radioquiet/radio-loud dichotomy in low excitation AGN.

Furthermore, if indeed galaxies similar with respect to their nuclear properties give rise to radio-sources of widely different power and morphology, the use of the total radio power as calorimeter of the jet is questioned. This also raises the issue how to define the radio-loudness of AGN. Apparently, RL and RQ AGN are best separated considering their nuclear properties, in particular by comparing the luminosity in the radio with X-ray and optical data (e.g. Capetti \& Balmaverde 2005), stressing again the importance of high-resolution multi-band measurements of their nuclear emission.

Finally, we note that an important issue in the study of these radio sources that are of generally low power is related to the identification of the dominant process of the radio emission, i.e. AGN or star formation. Best et al. (2005b) adopted a separation based on the radio luminosity expected for a stellar population of a given age (derived from the value of the $D_{n}(4000)$ index) and mass. While the proposed method is sufficiently accurate to build, e.g., radio luminosity functions, it cannot be safely applied to individual galaxies. Indeed, we found that many objects located just above the threshold in the $D_{n}(4000)$ vs. $L_{1.4 \mathrm{GHz}} / M_{*}$ plane have spectro-photometric characteristics very different from the bulk of the sample. This suggests that the "grey region" where the two populations (with radio powered by an AGN or by star formation) overlap covers a rather broad range of these parameters. This is likely due to the different scales probed by NVSS images and SDSS spectra. For example, we found a spiral galaxy whose SDSS spectrum is indicative of an old stellar population. However, the SDSS fiber covers only its bulge and, consequently, the predicted radio emission from star formation is relatively low, suggesting an AGN origin. However, no radio core is seen in the FIRST images, suggesting that the radio emission might originate from young stars in the spiral arms, which are not covered by the nuclear spectrum. Deeper radio images with a better resolution than the FIRST and NVSS maps could be used to isolate these objects based on their radio morphology and on the spatial association of radio and optical structures.

Acknowledgements. The authors acknowledge partial financial support from ASI grant I/023/050. This research has made use of the NASA/IPAC Extragalactic Database (NED) (which is operated by the Jet Propulsion Laboratory, California Institute of Technology, under contract with the National Aeronautics and Space Administration), of the LEDA database, and of the NASA's Astrophysics Data System (ADS). The research made use of the SDSS Archive, funding for the creation and distribution of which was provided by the Alfred P. Sloan Foundation, the Participating Institutions, the National Aeronautics and Space Administration, the National Science Foundation, the US Department of Energy, the Japanese Monbukagakusho, and the Max Planck Society, and The Higher Education Funding Council for England. The research uses the NVSS and FIRST radio surveys, carried out using the NRAO Very Large Array: NRAO is operated by Associated Universities Inc., under co-operative agreement with the National Science Foundation.

\section{References}

Baldi, R., Chiaberge, M., Capetti, A., Macchetto, F., \& Sparks, W. 2010, ApJ, submitted

Baldi, R. D., \& Capetti, A. 2008, A\&A, 489, 989

Baldi, R. D., \& Capetti, A. 2009, A\&A, 508, 603

Baldwin, J. A., Phillips, M. M., \& Terlevich, R. 1981, PASP, 93, 5

Balmaverde, B., \& Capetti, A. 2006, A\&A, 447, 97

Balogh, M. L., Morris, S. L., Yee, H. K. C., Carlberg, R. G., \& Ellingson, E. 1999, ApJ, 527, 54

Baum, S. A., \& Heckman, T. 1989, ApJ, 336, 702

Becker, R. H., White, R. L., \& Helfand, D. J. 1995, ApJ, 450, 559

Bell, E. F., McIntosh, D. H., Katz, N., \& Weinberg, M. D. 2003, ApJS, 149, 289

Bell, E. F., Zheng, X. Z., Papovich, C., et al. 2007, ApJ, 663, 834

Bernardi, M., Sheth, R. K., Annis, J., et al. 2003, AJ, 125, 1849

Bernardi, M., Shankar, F., Hyde, J. B., et al. 2010, MNRAS, 404, 2087

Best, P. N., Kauffmann, G., Heckman, T. M., et al. 2005a, MNRAS, 362, 25

Best, P. N., Kauffmann, G., Heckman, T. M., \& Ivezić, Ž. 2005b, MNRAS, 362, 9 
Buttiglione, S., Capetti, A., Celotti, A., et al. 2009, A\&A, 495, 1033 Buttiglione, S., Capetti, A., Celotti, A., et al. 2010, A\&A, 509, A6 Capetti, A., \& Balmaverde, B. 2005, A\&A, 440, 73

Chiaberge, M. 2008, in COSPAR, 37th COSPAR Scientific Assembly, Plenary Meeting, 37, 532

Cid Fernandes, R., Stasinska, G., Vale Asari, N., et al. 2010, IAU Symp., 267, 65

Condon, J. J., Cotton, W. D., Greisen, E. W., et al. 1998, AJ, 115, 1693

Heckman, T. M. 1980, A\&A, 87, 152

Heinz, S., Merloni, A., \& Schwab, J. 2007, ApJ, 658, L9

Hine, R. G., \& Longair, M. S. 1979, MNRAS, 188, 111

Hyde, J. B., \& Bernardi, M. 2009, MNRAS, 394, 1978

Jackson, N., \& Rawlings, S. 1997, MNRAS, 286, 241

Kauffmann, G., Heckman, T. M., White, S. D. M., et al. 2003, MNRAS, 341, 33

Kewley, L. J., Groves, B., Kauffmann, G., \& Heckman, T. 2006, MNRAS, 372, 961

Labiano, A. 2008, A\&A, 488, L59

Laing, R. A., Jenkins, C. R., Wall, J. V., \& Unger, S. W. 1994, in The First Stromlo Symposium: The Physics of Active Galaxies, ed. G. V. Bicknell, M. A. Dopita, \& P. J. Quinn, ASP Conf. Ser., 54, 201

Lintott, C. J., Schawinski, K., Slosar, A., et al. 2008, MNRAS, 389, 1179

Madrid, J. P., Chiaberge, M., Floyd, D., et al. 2006, ApJS, 164, 307
Mannucci, F., Basile, F., Poggianti, B. M., et al. 2001, MNRAS, 326, 745

Marconi, A., \& Hunt, L. K. 2003, ApJ, 589, L21

Martel, A. R., Baum, S. A., Sparks, W. B., et al. 1999, ApJS, 122, 81

Martin, P. G., Angel, J. R. P., \& Maza, J. 1976, ApJ, 209, L21

Morganti, R., Tadhunter, C. N., Dickson, R., \& Shaw, M. 1997, A\&A, 326, 130

Nakamura, O., Fukugita, M., Yasuda, N., et al. 2003, AJ, 125, 1682

O'Dea, C. P., Koekemoer, A. M., Baum, S. A., et al. 2001, AJ, 121, 1915

Prandoni, I., de Ruiter, H. R., Ricci, R., et al. 2010, A\&A, 510, A42

Rawlings, S., Saunders, R., Eales, S. A., \& Mackay, C. D. 1989, MNRAS, 240, 701

Schawinski, K., Lintott, C., Thomas, D., et al. 2009, MNRAS, 396, 818

Shen, S., Mo, H. J., White, S. D. M., et al. 2003, MNRAS, 343, 978

Smolčić, V. 2009, ApJ, 699, L43

Stoughton, C., Lupton, R. H., Bernardi, M., et al. 2002, AJ, 123, 485

Strateva, I., Ivezić, Ž., Knapp, G. R., et al. 2001, AJ, 122, 1861

Tadhunter, C. N., Morganti, R., Robinson, A., et al. 1998, MNRAS, 298, 1035

Tremaine, S., Gebhardt, K., Bender, R., et al. 2002, ApJ, 574, 740

Whittle, M. 1985, MNRAS, 213, 33

Willott, C. J., Rawlings, S., Blundell, K. M., \& Lacy, M. 1999, MNRAS, 309, 1017

York, D. G., Adelman, J., Anderson, Jr., J. E., et al. 2000, AJ, 120, 1579

Pages 15 to 17 are available in the electronic edition of the journal at http://www. aanda.org 


\section{Appendix A: Appendix}

We report in this Appendix the analogous of Fig. 1 (the spectroscopic diagnostic diagrams), Fig. 2 (left panel) (H $\alpha$ flux vs. the continuum at the $\mathrm{H} \alpha$ line), Fig. 3 (concentration index vs. $4000 \AA$ break), Fig. $6\left(D_{n}(4000)\right.$ vs. $\left.L_{1.4 \mathrm{GHz}} / M_{*}\right)$, Fig. 9 (distributions of the SMBH masses for each spectroscopic class), and Fig. 7 (total radio power vs. [O III] luminosities) for the objects with redshift $0.1<z<0.3$.

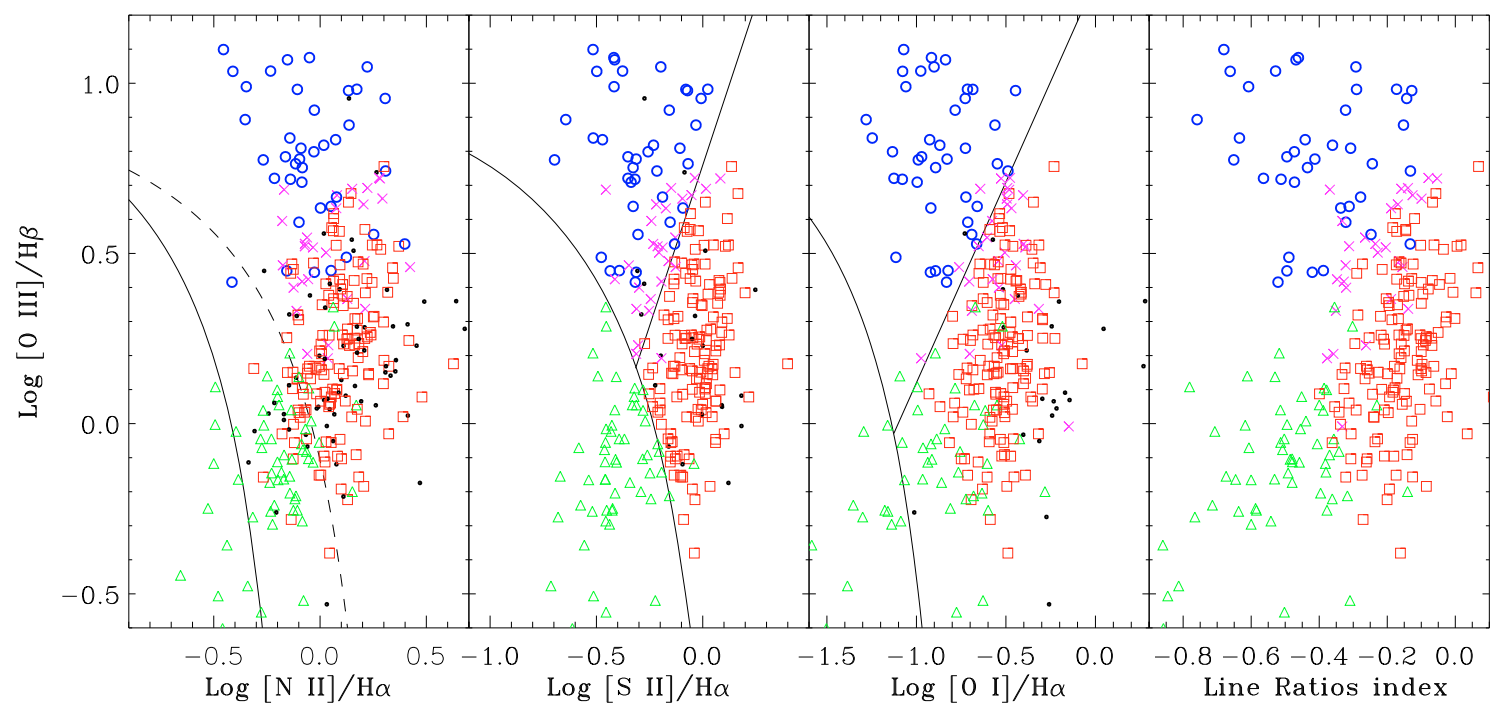

Fig. A.1. Spectroscopic diagnostic diagrams for the objects with $0.1<z<0.3$, with the same structure and symbols as Fig. 1. 
A\&A 519, A48 (2010)
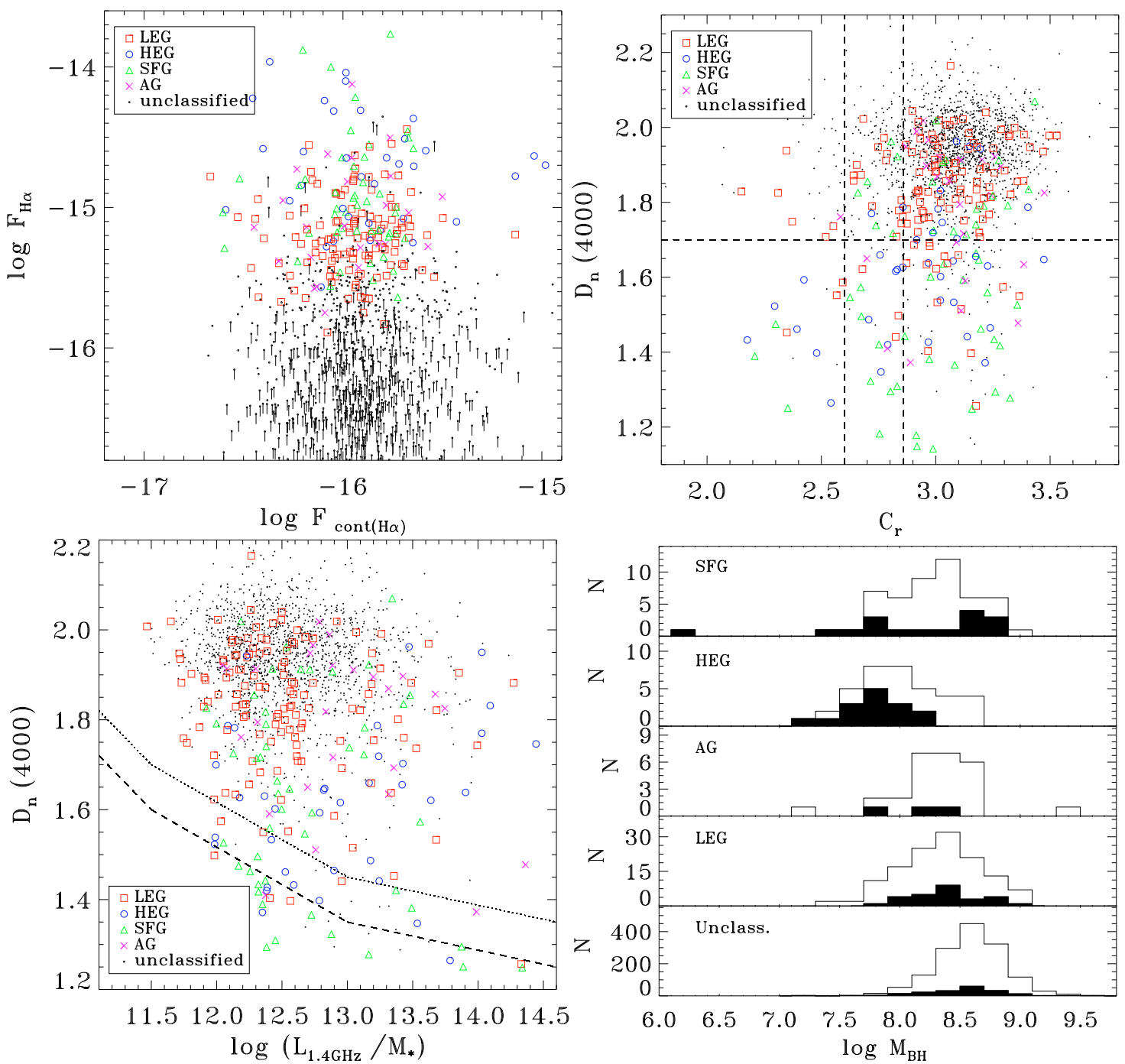

Fig. A.2. Analogous of Fig. 2 (left panel), Figs. 3, 6, and 9 for the higher redshift objects $(0.1<z<0.3)$ i.e.: (upper left panel) logarithm of the $\mathrm{H} \alpha$ flux versus logarithm of the continuum at the $\mathrm{H} \alpha$ line (in erg s${ }^{-1}$ and $\operatorname{erg~s}^{-1} \AA^{-1}$ units respectively); (upper right panel) concentration index $C_{r}$ vs. $4000 \AA$ break; (lower left panel) $D_{n}(4000)$ vs. $L_{1.4 \mathrm{GHz}} / M_{*}$; (lower right panel) distributions of the $M_{\mathrm{BH}}$ (in solar units) for the various spectroscopic classes. The filled portion of the histograms represent the contribution of galaxies with $C_{r}<2.86$. 
R. D. Baldi and A. Capetti: Spectro-photometric properties of the radio-loud AGN population

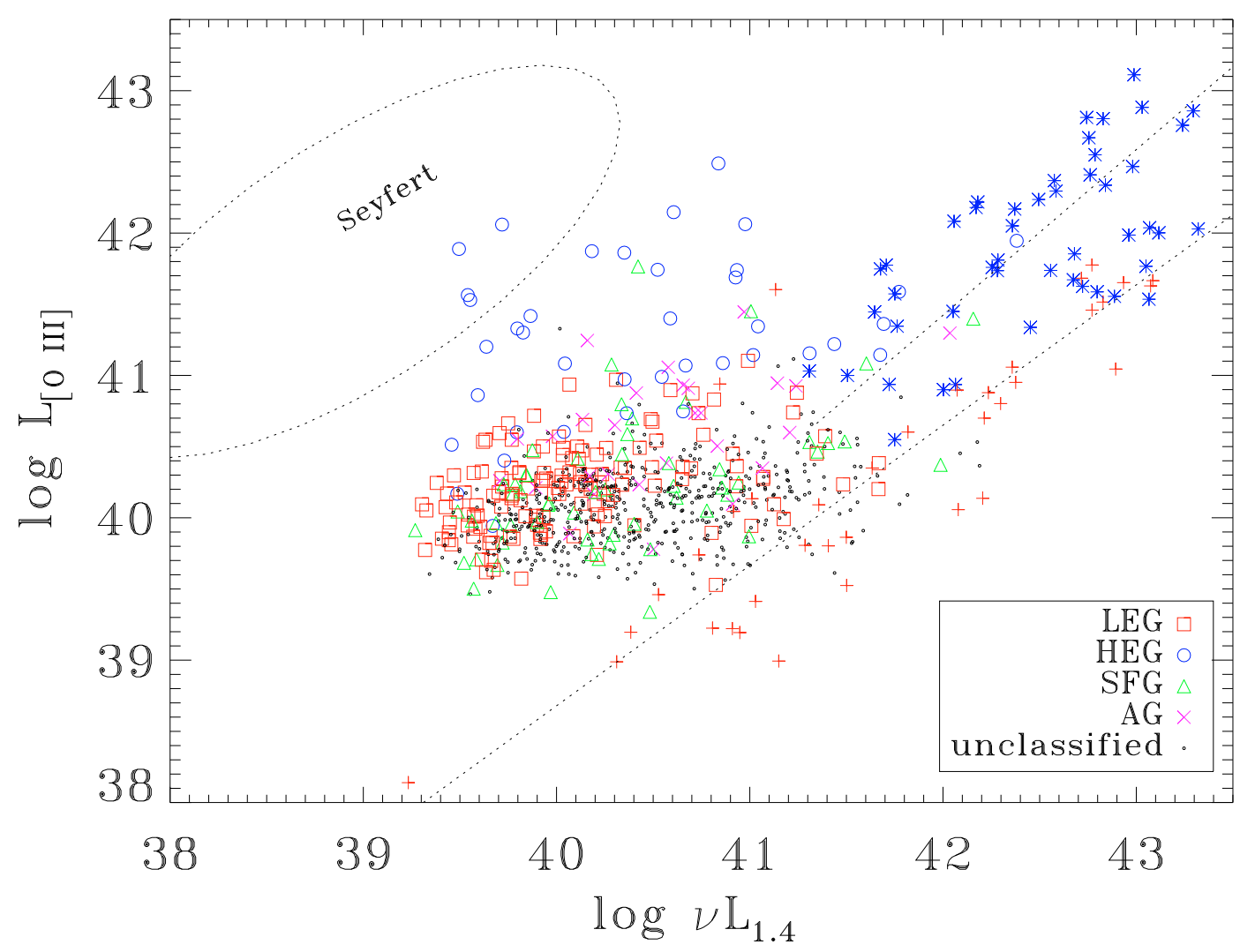

Fig. A.3. Analogous of Fig. 7 for the higher redshift objects $(0.1<z<0.3)$ i.e. logarithm of radio power $L_{1.4 \mathrm{GHz}}$ Vs. [O III] luminosities. For more clarity the large number of upper limits in $\left.L_{[\mathrm{O}} \mathrm{III}\right]$ are not shown. The two dashed lines reproduce the line-radio correlation followed by the LEG and HEG of the 3CR sample (red plusses and blue asterisks respectively). The ellipse marks the boundaries of the location of Seyfert galaxies (e.g. Whittle 1985). 